TRANSACTIONS OF THE

AMERICAN MATHEMATICAL SOCIETY

Volume 352, Number 12, Pages 5817-5854

S 0002-9947(00)02565-4

Article electronically published on June 21, 2000

\title{
INTERSECTION THEORY ON NON-COMMUTATIVE SURFACES
}

\author{
PETER JØRGENSEN
}

\begin{abstract}
Consider a non-commutative algebraic surface, $X$, and an effective divisor $Y$ on $X$, as defined by Van den Bergh. We show that the Riemann-Roch theorem, the genus formula, and the self intersection formula from classical algebraic geometry generalize to this setting.

We also apply our theory to some special cases, including the blow up of $X$ in a point, and show that the self intersection of the exceptional divisor is -1 . This is used to give an example of a non-commutative surface with a commutative $\mathbb{P}^{1}$ which cannot be blown down, because its self intersection is +1 rather than -1 . We also get some results on Hilbert polynomials of modules on $X$.
\end{abstract}

\section{INTRODUCTION}

In any decent world, two curves on a surface should have an intersection. This is so fundamental that it ought to hold even when "curve" and "surface" mean something other than they normally do.

The above truism has become topical with Van den Bergh's paper [10], which introduces a new general framework for the study of non-commutative algebraic surfaces, and curves and points on such surfaces. Van den Bergh's setup is so comprehensive, including things such as blow up, that we can hope to develop a theory of non-commutative surfaces which parallels the commutative theory closely. Hence it seems obvious that we should try to develop a non-commutative intersection theory - after all, intersection theory is one of the essential ingredients in the classical commutative theory of algebraic surfaces. This project has been initiated in [7], 8], and [9].

Also, it is hoped that the theory of non-commutative surfaces will help us to understand 3-dimensional graded algebras better: When $A$ is such an algebra, $\operatorname{Proj}(A)$ (as constructed in [2]) is a non-commutative algebraic surface (and this is the principal way of getting such surfaces). Again, intersection theory should prove a useful ingredient, just as in the classical commutative theory.

This manuscript does some intersection theory within the framework set up by Van den Bergh in 10 to which we refer for the terminology of "quasi-schemes" which we will employ, but see also section 1 below for an explanation of some rudiments of the theory. However, for the purpose of this introduction, one should just think of quasi-schemes as some sort of "non-commutative schemes", and keep

Received by the editors June 16, 1998 and, in revised form, March 31, 1999.

2000 Mathematics Subject Classification. Primary 14A22, 16W50.

Key words and phrases. Quasi-scheme, effective divisor, intersection multiplicity, non-commutative surface, non-commutative Riemann-Roch theorem, non-commutative blow up.

(C)2000 American Mathematical Society 
in mind that a special case of a non-commutative scheme is a commutative scheme — in other words, an ordinary scheme!

The strategy we will use for getting intersection theory is the following: We start with a quasi-scheme $\left(X, \mathcal{O}_{X}\right)$ over the field $k$. We suppose that $Y$ is an effective divisor on $X$. We then define $c(Y)$, the first Chern class of $Y$, as the operator

$$
[M] \longmapsto[M]-[M(-Y)]
$$

defined on the $K$-theory of $X$, which we use as a substitute for $X$ 's (presumably non-existing) Chow groups. We think of $c(Y)$ as being the operator "intersect a $K$ class with the hypersurface determined by the divisor $Y$ ". This idea of defining the first Chern class as an operator on $K$-theory, rather than an element in $K$-theory (as many authors do) is borrowed from [3]. The operator point of view turns out to be very well suited to the present situation.

The Chern class $c(Y)$ is now used to define intersection multiplicities: If $C \in$ $\bmod (X)$ is a curve module on the quasi-scheme $X$, we define the intersection multiplicity between the divisor $Y$ and the curve $C$ by

$$
\langle Y,[C]\rangle=\chi(c(Y)([C])),
$$

where $\chi$ is Euler characteristic, which is well-defined on $K$-theory.

Specializing the above to the case where $X$ is 2-dimensional (i.e. a "quantum surface"), and making a number of further assumptions on $X$ and $Y$ (which basically say that $X$ is proper, and also prohibit the worst pathological behaviour), it turns out that one can prove several theorems which directly generalize well-known results from the intersection theory of commutative projective surfaces:

- There is a Riemann-Roch theorem, theorem 5.1, stating that

$$
\chi\left(\mathcal{O}_{X}(Y)\right)=\chi\left(\mathcal{O}_{X}\right)+\frac{1}{2}\left(\left\langle Y,\left[\mathcal{O}_{Y}\right]\right\rangle-\left\langle Y,[\omega]-\left[\mathcal{O}_{X}\right]\right\rangle\right) .
$$

For a detailed explanation of the symbols involved in this formula, we refer to sections 3 and 4 below. However, note already here that, typographically speaking, the terms in the Riemann-Roch formula look like terms well-known from commutative algebraic geometry, and that, when $X$ is in fact a commutative projective algebraic surface, the terms in the formula become equal to the relevant terms from commutative geometry.

- There is a genus formula, theorem[5.2] stating that

$$
2 g(Y)-2=\left\langle Y,\left[\mathcal{O}_{Y}\right]\right\rangle+\left\langle Y,[\omega]-\left[\mathcal{O}_{X}\right]\right\rangle,
$$

where $g(Y)$ is the "genus" of the "non-commutative curve" $Y$. Note that if $Y$ is in fact a commutative smooth projective curve, then $g(Y)$ is the usual genus of $Y$.

- There is a self intersection formula, theorem 4.5, stating that

$$
\left\langle Y,\left[\mathcal{O}_{Y}\right]\right\rangle=\operatorname{deg}(\mathcal{N}),
$$

where $\mathcal{N}$ is the normal bundle of $Y$ in $X$.

- If $p \in X$ is a point, and $\alpha: \widetilde{X} \longrightarrow X$ is the blow up of $X$ in $p$, and if $p$ is such that the blow up admits an exceptional divisor, $E$ (see [10, sec. 6.6]), then on $\tilde{X}$, by theorem 6.3 we have that

$$
\left\langle E,\left[\mathcal{O}_{E}\right]\right\rangle=-1
$$


In fact, all these results contain the corresponding commutative results as special cases.

We also give some sample applications of these results. First, we develop a theory of Hilbert polynomials of modules on non-commutative surfaces. The principal results here are corollary 5.3 theorem 5.6, and corollary 5.9. Secondly, we perform some concrete computations. One such is example 5.5. where Riemann-Roch is employed to compute the degree of the normal bundle of a certain effective divisor on an elliptic quantum $\mathbb{P}^{2}$. Another is example 6.4, where Riemann-Roch is applied to a certain quantum $\mathbb{P}^{2}$, which contains an effective divisor isomorphic to $\mathbb{P}^{1}$. Riemann-Roch here tells us that the divisor has self intersection different from -1 , and so cannot be blown down.

The results of this paper can be used on non-commutative surfaces of the form $\operatorname{Proj}(A)$ where $A$ is a 3 -dimensional graded algebra, illustrating the point made above that the theory of non-commutative algebraic surfaces can be applied to get results about such algebras.

One general remark: The structure of the theory in this paper differs from commutative geometry in one important way. In the commutative theory, there is an isomorphism between the group of divisor classes, $\operatorname{Pic}(X)$, and a certain slice of $K(X)$, namely

$$
\operatorname{Pic}(X) \cong F_{d-1} K(X) / F_{d-2} K(X)
$$

(the filtration $F_{i} K(X)$ of $K(X)$ is given by dimension of support). In the noncommutative theory, this breaks down: Now $\operatorname{Pic}(X)$, the group of divisor classes, consists of certain bi-modules in the sense of [10] (see section [1 below), and could be a non-commutative group. And there is no reason to suppose that an arbitrarily chosen element of

$$
F_{d-1} K(X) / F_{d-2} K(X)
$$

has a bi-module corresponding to it; presumably, there are only rather few bimodules. In other words, there is a genuine schism between "one-sided divisors" (given by elements of $F_{d-1} K(X) / F_{d-2} K(X)$ ) and "two-sided divisors" (given by bi-modules), and this is reflected in the design of the intersection theory, which uses both bi-modules and (classes of) elements of $K(X)$.

Of course, one has to prove a number of things about $c(Y)$ and $\langle Y,-\rangle$ in order to get the results quoted. The manuscript is therefore structured as follows: Section 1 collects a number of definitions and basic lemmas concerning quasi-schemes and effective divisors on quasi-schemes. Section 2 defines positive multiples of effective divisors, and gives some technical results; it turns out that positive multiples are handy when combined e.g. with the Riemann-Roch theorem. Section 3 defines $c(Y)$ and proves some properties. Section 4 defines $\langle Y,-\rangle$ and proves some properties, and also proves the self intersection formula. Section 5 contains the Riemann-Roch theorem, the genus formula, and some consequences, mainly for Hilbert polynomials. Finally, section 6 applies the theory to the case of a non-commutative blow up, and proves the result about the exceptional divisor quoted above.

\section{ACKNOWLEDGEMENT}

I must express my appreciation of discussions with Paul Smith, whose vision of non-commutative geometry enlightens this entire project.

In the fall of 1998 I was a post doc. in Antwerp, financed by the TMR-network "Algebraic Lie Representations". 


\section{EFFECTIVE DIVISORS ON QUASI-SCHEMES}

Throughout this manuscript, $k$ is a fixed field. All categories and functors are $k$ linear.

This section collects a number of definitions and basic properties from the theory of quasi-schemes as laid out in [10]. It deals with the following subjects:

- Quasi-schemes and bi-modules on quasi-schemes (definitions 1.1 and 1.2).

- Effective divisors and their basic properties (definition 1.3 to example 1.10).

- The $K$-theory of quasi-schemes (definition 1.11 to definition 1.13).

- Useful conditions which can be imposed on effective divisors (definition 1.14).

- The case of a commutative divisor (proposition 1.15).

When reading below, one should keep in mind that commutative schemes, divisors and so on are special cases of the non-commutative objects introduced. For this to make sense, one has to identify a commutative scheme $X$ with the Grothendieck category $\operatorname{Mod}(X)$ of quasi-coherent sheaves on $X$, and identify an invertible sheaf $\mathcal{L}$ with the functor $\mathcal{H o m}(\mathcal{L},-)$.

We start by reiterating from 10 the notion of quasi-scheme and the definition of a bi-module on a quasi-scheme.

Definition 1.1. A quasi-scheme over $k$ is a pair $\left(X, \mathcal{O}_{X}\right)$, where $X$ is a $k$-linear Grothendieck category, $\mathcal{O}_{X}$ an object of $X$.

We follow [10] and define $\operatorname{Mod}(X)=X$.

$($ For $\operatorname{Mod}(X)$ to be a Grothendieck category means that it is abelian, cocomplete, that colimits over small directed sets are exact in $\operatorname{Mod}(X)$, and that $\operatorname{Mod}(X)$ has a small set of generators. Note that a Grothendieck category has enough injectives; in fact, it has injective envelopes.)

If $\mathcal{O}_{X}$ is a noetherian object of $\operatorname{Mod}(X)$, and $\operatorname{Mod}(X)$ has a small set of generators which are noetherian, then we call the quasi-scheme $\left(X, \mathcal{O}_{X}\right)$ over $k$ noetherian.

In the noetherian case, we write $\bmod (X)$ for the full subcategory of $\operatorname{Mod}(X)$ which consists of all noetherian objects.

Definition 1.2. Let $\left(X, \mathcal{O}_{X}\right)$ be a quasi-scheme over $k$.

We write

$$
\operatorname{BIMOD}(X, X)=\operatorname{LeftExact}_{k}(\operatorname{Mod}(X), \operatorname{Mod}(X))^{\text {opp }},
$$

where LeftExact $k$ (A, B) denotes the category of $k$-linear left exact functors from A to $\mathrm{B}$, when $\mathrm{A}, \mathrm{B}$ are $k$-linear categories.

If $\mathcal{M}$ is an object of $\operatorname{BIMOD}(X, X)$, we write

$$
\mathcal{H o m}(\mathcal{M},-)
$$

for the corresponding functor in $\operatorname{LeftExact}_{k}(\operatorname{Mod}(X), \operatorname{Mod}(X))$. Composition of functors is denoted by $\otimes$, so

$$
\mathcal{H o m}(\mathcal{M} \otimes \mathcal{N},-)=\mathcal{H o m}(\mathcal{M},-) \circ \mathcal{H o m}(\mathcal{N},-)=\mathcal{H o m}(\mathcal{M}, \mathcal{H o m}(\mathcal{N},-)) .
$$
So

The identity functor on $\operatorname{Mod}(X)$ gives us a bi-module which we denote by $o_{X}$.

$$
\mathcal{H o m}\left(o_{X},-\right)=\operatorname{id}_{\operatorname{Mod}(X)} .
$$

A bi-module $\mathcal{M}$ is called invertible if the functor $\mathcal{H o m}(\mathcal{M},-)$ is an autoequivalence. 
Finally, we also write

$$
\operatorname{Bimod}(X, X)=\left\{\mathcal{M} \in \operatorname{BIMOD}(X, X) \mid \mathcal{H}_{\text {om }}(\mathcal{M},-) \text { has a left adjoint }\right\}
$$

Note that by 10 , prop. 3.1.1(3)], the category $\operatorname{BIMOD}(X, X)$ is abelian, and in fact $k$-linear.

We go on to discuss effective divisors on a quasi-scheme.

Definition 1.3. Let $\left(X, \mathcal{O}_{X}\right)$ be a quasi-scheme over $k$. An effective divisor $Y$ on $X$ is a subobject of $o_{X}$ (in the category $\operatorname{BIMOD}(X, X)$ ) for which each representing monic, $\mathcal{I} \hookrightarrow o_{X}$, satisfies that the bi-module $\mathcal{I}$ is invertible.

Any $\operatorname{BIMOD}(X, X)$-object which represents the subobject $Y$ is denoted $o_{X}(-Y)$. So $o_{X}(-Y)$ comes with a monic $o_{X}(-Y) \hookrightarrow o_{X}$, and $o_{X}(-Y)$ is invertible and determined up to unique isomorphism.

It should be noted that the word "subobject" in this definition is meant in the strict categorical sense; see [6. p. 122]. So the definition in fact says that an effective divisor $Y$ is a certain class of monics going into $o_{X}$. The use of the notation $o_{X}(-Y)$ is possible because it will always be true that only the $\operatorname{BIMOD}(X, X)$-isomorphism class of $o_{X}(-Y)$ really matters. Of course, this class is the same no matter which representing object of $Y$ we take. Also for this reason, we usually write "=" between bi-modules even when we only mean that they are isomorphic, and not literally equal.

In the next definition, our conventions can be seen at work.

Definition 1.4. Let $\left(X, \mathcal{O}_{X}\right)$ be a quasi-scheme over $k$, let $Y$ be an effective divisor on $X$, and let $n$ be an integer.

We write

$$
o_{X}(n Y)=\left(o_{X}(-Y)\right)^{\otimes(-n)}
$$

(where it is understood that raising to a negative power is defined by raising the inverse bi-module to the corresponding positive power).

For $M \in \operatorname{Mod}(X)$ we write

$$
M(n Y)=M \otimes o_{X}(n Y)
$$

(see [10. secs. 3.1 and 3.7] for a discussion of this notation, which is based on identifying $M$ with the bi-module given by the functor $\operatorname{Hom}_{X}(M,-)$. Note that $\left.\mathcal{H o m}\left(o_{X}(n Y), M\right)=M(-n Y)\right)$.

Some remarks: The bi-module $o_{X}(n Y)$ is only defined up to isomorphism. This is because, as noted above, the bi-module $o_{X}(-Y)$ itself is only defined up to isomorphism, since it is only defined as a representative of an $o_{X}$-subobject.

If $Y$ is an effective divisor on $X$, we will see in section 2 that $o_{X}(-n Y)$ determines an effective divisor for any $n \geq 0$.

Note that invertibility of $\mathcal{H}^{\circ} m_{X}\left(o_{X}(-Y),-\right)$ implies that $o_{X}(-Y)$ is in $\operatorname{Bimod}(X, X)$. As a consequence of the following lemma, we see that $o_{X} / o_{X}(-Y)$ is also in $\operatorname{Bimod}(X, X)$.

Lemma 1.5. Let $\left(X, \mathcal{O}_{X}\right)$ be a quasi-scheme over $k$, and let

$$
0 \rightarrow \mathcal{M}^{\prime} \longrightarrow \mathcal{M} \longrightarrow \mathcal{M}^{\prime \prime} \rightarrow 0
$$

be an exact sequence in $\operatorname{BIMOD}(X, X)$.

If $\mathcal{M}^{\prime}$ and $\mathcal{M}$ are in $\operatorname{Bimod}(X, X)$, then also $\mathcal{M}^{\prime \prime}$ is in $\operatorname{Bimod}(X, X)$. 
Proof. By [10, prop. 3.1.1(5)], we need to see that $\mathcal{H}$ om $\left(\mathcal{M}^{\prime \prime},-\right)$ commutes with direct products when evaluated on injectives. For the purpose of this proof only, we dispense with the $\mathcal{H} o m$-notation, and write $\mathcal{M}^{\prime \prime}(M)$ rather than $\mathcal{H} o m\left(\mathcal{M}^{\prime \prime}, M\right)$.

So let $\left\{I_{\alpha}\right\}_{\alpha}$ be a small family of injectives from $\operatorname{Mod}(X)$. We have a commutative diagram

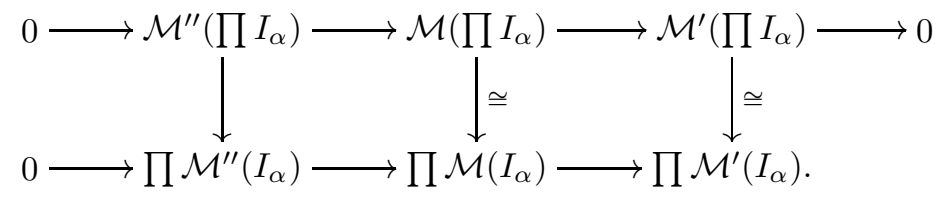

The top row is exact since $\prod I_{\alpha}$ is injective (cf. [10, proof of 3.1.1(3)]). Similarly, each sequence

$$
0 \rightarrow \mathcal{M}^{\prime \prime}\left(I_{\alpha}\right) \longrightarrow \mathcal{M}\left(I_{\alpha}\right) \longrightarrow \mathcal{M}^{\prime}\left(I_{\alpha}\right) \rightarrow 0
$$

is exact, and since $\prod$ is a left exact functor, this means that the diagram's bottom row is exact. Finally, the two rightmost vertical morphisms in the diagram are isomorphisms by [10 prop. 3.1.1(5)], since we have assumed $\mathcal{M}, \mathcal{M}^{\prime} \in \operatorname{Bimod}(X, X)$.

Applying the five lemma to the diagram, we now get what we want: The canonical map

$$
\mathcal{M}^{\prime \prime}\left(\prod I_{\alpha}\right) \longrightarrow \prod \mathcal{M}^{\prime \prime}\left(I_{\alpha}\right)
$$

is an isomorphism.

Definition 1.6. Let $\mathrm{A}$ be a $k$-linear category with a full subcategory $\mathrm{A}^{\prime}$. Write $i_{*}: \mathrm{A}^{\prime} \hookrightarrow \mathrm{A}$ for the embedding functor.

We say that $A^{\prime}$ is closed in $A$ if $A^{\prime}$ is closed under subquotients (taken in $A$ ), and the functor $i_{*}$ has a right adjoint.

We say that $A^{\prime}$ is bi-closed in $A$ if $A^{\prime}$ is closed under subquotients (taken in $A$ ), and the functor $i_{*}$ has both a right and a left adjoint.

Proposition 1.7. Let $\left(X, \mathcal{O}_{X}\right)$ be a quasi-scheme over $k$, and let $Y$ be an effective divisor on $X$ determined by $o_{X}(-Y) \hookrightarrow o_{X}$. Write $\lambda$ : id $\longrightarrow \mathcal{H}$ om $\left(o_{X}(-Y),-\right)$ for the corresponding natural transformation.

By [10, prop. 3.4.2], the $\operatorname{BIMOD}(X, X)$-monic $o_{X}(-Y) \hookrightarrow o_{X}$ determines a category, $\operatorname{Mod}\left(o_{X} / o_{X}(-Y)\right)$, which can be viewed as a full bi-closed subcategory of $\operatorname{Mod}(X)$ (bi-closedness follows since $o_{X} / o_{X}(-Y) \in \operatorname{Bimod}(X, X)$ by lemma 1.5). We denote the embedding functor by

$$
i_{*}: \operatorname{Mod}\left(o_{X} / o_{X}(-Y)\right) \hookrightarrow \operatorname{Mod}(X) .
$$

$\left.1^{\circ}\right)$ The right-adjoint of $i_{*}$ is given by

$$
i^{!}(-)=\mathcal{H o m}\left(o_{X} / o_{X}(-Y),-\right),
$$

and the left-adjoint of $i_{*}$ is given by

$$
i^{*}(-)=-\otimes o_{X} / o_{X}(-Y)=\mathcal{H o m}\left(o_{X}(-Y)^{-1}, R^{1} i^{!}(-)\right) .
$$

$\left.2^{\circ}\right)$ The pair

$$
\left(\operatorname{Mod}\left(o_{X} / o_{X}(-Y)\right), i^{*}\left(\mathcal{O}_{X}\right)\right)
$$

is a quasi-scheme over $k$. If $\left(X, \mathcal{O}_{X}\right)$ is noetherian, then so is the pair (1.1).

$\left.3^{\circ}\right)$ For any $M \in \operatorname{Mod}(X)$, there is an exact sequence

$$
0 \rightarrow i^{!}(M) \longrightarrow M \stackrel{\lambda_{M}}{\longrightarrow} \mathcal{H o m}\left(o_{X}(-Y), M\right) \longrightarrow R^{1} i^{!}(M) \rightarrow 0 .
$$


$\left.4^{\circ}\right)$ If $M \in \operatorname{Mod}(X)$, then

$$
M \in \operatorname{Mod}\left(o_{X} / o_{X}(-Y)\right) \text { if and only if } \lambda_{M}=0 .
$$

$\left.5^{\circ}\right)$ The subcategory $\operatorname{Mod}\left(o_{X} / o_{X}(-Y)\right)$ of $\operatorname{Mod}(X)$ is closed under the functors $\mathcal{H o m}\left(o_{X}(-Y),-\right)$ and $\mathcal{H o m}\left(o_{X}(-Y)^{-1},-\right)=-\otimes o_{X}(-Y)$. So these functors define inverse autoequivalences of

$$
\operatorname{Mod}\left(o_{X} / o_{X}(-Y)\right) \text {. }
$$

Proof. $1^{\circ}$ : The formulas for the right and left adjoints of $i_{*}$ follow from the discussion in [10 beginning of sec. 3.4] (one has to compute a bit to see $i^{*}(-)=$ $\left.\mathcal{H o m}\left(o_{X}(-Y)^{-1}, R^{1} i^{!}(-)\right)\right)$.

$3^{\circ}$ : The short exact sequence

$$
0 \rightarrow o_{X}(-Y) \longrightarrow o_{X} \longrightarrow o_{X} / o_{X}(-Y) \rightarrow 0
$$

in $\operatorname{BIMOD}(X, X)$ corresponds to a short exact sequence

$$
0 \rightarrow \mathcal{H o m}\left(o_{X} / o_{X}(-Y),-\right) \longrightarrow \mathcal{H o m}\left(o_{X},-\right) \longrightarrow \mathcal{H o m}\left(o_{X}(-Y),-\right) \rightarrow 0
$$

in $\operatorname{LeftExact}_{k}(\operatorname{Mod}(X), \operatorname{Mod}(X))$. Rewriting, this is

$$
0 \rightarrow i^{!} \longrightarrow \mathrm{id} \longrightarrow \mathcal{H} \text { om }\left(o_{X}(-Y),-\right) \rightarrow 0 \text {. }
$$

If one applies this sequence to an injective object from $\operatorname{Mod}(X)$, one gets a short exact sequence in $\operatorname{Mod}(X)$ (see [10 proof of 3.1.1(3)]), and consequently, for any $M \in \operatorname{Mod}(X)$, there is a long-exact sequence of right-derived functors, consisting of pieces

$$
R^{j} i^{!}(M) \longrightarrow R^{j} \operatorname{id}(M) \longrightarrow \mathcal{E} x t^{j}\left(o_{X}(-Y), M\right),
$$

where we use the obvious notation $\mathcal{E} x t^{j}=\mathrm{R}^{j} \mathcal{H}$ om. The start of this sequence gives the sequence (1.2).

$4^{\circ}$ : It is easy to see that if $M \in \operatorname{Mod}(X)$, then $i^{!}(M)$ is equal to the maximal $M$-subobject which is in $\operatorname{Mod}\left(o_{X} / o_{X}(-Y)\right)$. So we have

$$
M \in \operatorname{Mod}\left(o_{X} / o_{X}(-Y)\right) \Leftrightarrow i^{!}(M) \longrightarrow M \text { is an isomorphism } \Leftrightarrow \lambda_{M}=0,
$$

where the second " $\Leftrightarrow$ " follows from the sequence (1.2).

Note that this result shows that we are in fact in the situation described in [11. sec. 8]. Our notation can be translated into the notation of [11 as follows:

$\begin{array}{cc}\frac{\text { our notation }}{\operatorname{Mod}(X)} & \frac{\text { 11]'s notation }}{\mathcal{A}} \\ \mathcal{H} \operatorname{Hom}\left(o_{X}(-Y),-\right) & G^{-1} \\ \operatorname{Mod}\left(o_{X} / o_{X}(-Y)\right) & \mathcal{B} \\ \lambda & G^{-1}(\eta) .\end{array}$

$2^{\circ}$ : It is clear from [11 lem. 8.1(1)] that $\operatorname{Mod}\left(o_{X} / o_{X}(-Y)\right)$ is cocomplete and has exact colimits over small directed sets. And it is easy to see that if $\left\{\mathcal{O}_{\alpha}\right\}_{\alpha}$ is a small set of generators of $\operatorname{Mod}(X)$, then

$$
\left\{i^{*} \mathcal{O}_{\alpha}\right\}_{\alpha}
$$

is a small set of generators of $\operatorname{Mod}\left(o_{X} / o_{X}(-Y)\right)$. So $\operatorname{Mod}\left(o_{X} / o_{X}(-Y)\right)$ is a Grothendieck category, and

$$
\left(\operatorname{Mod}\left(o_{X} / o_{X}(-Y)\right), i^{*}\left(\mathcal{O}_{X}\right)\right)
$$

is a quasi-scheme over $k$. 
And generally, if $M \in \operatorname{Mod}(X)$ is noetherian, then $i^{*}(M)$ is also noetherian, because there is an epimorphism $M \longrightarrow i^{*}(M)$. So suppose that $\left(X, \mathcal{O}_{X}\right)$ is noetherian. Then $\mathcal{O}_{X}$ is noetherian, and all the $\mathcal{O}_{\alpha}$ 's can be chosen noetherian. This makes $i^{*}\left(\mathcal{O}_{X}\right)$ and all the $i^{*}\left(\mathcal{O}_{\alpha}\right)$ noetherian, hence (1.3) is a noetherian quasischeme over $k$.

$5^{\circ}$ : As one can see using the above dictionary between our notation and [11's notation, this is proved as [11, lem. 8.1(2)].

Remark 1.8. If we are in the situation of proposition 1.7 and twist the exact sequence (1.2) by $-Y$, we get an exact sequence

$$
0 \rightarrow\left(i^{!} M\right)(-Y) \longrightarrow M(-Y) \stackrel{\lambda_{M}(-Y)}{\longrightarrow} M \longrightarrow i^{*} M \rightarrow 0,
$$

which is frequently handier than (1.2) itself.

Definition 1.9. Let $\left(X, \mathcal{O}_{X}\right)$ be a quasi-scheme over $k$, let $Y$ be an effective divisor on $X$, and denote by $i_{*}: \operatorname{Mod}\left(o_{X} / o_{X}(-Y)\right) \hookrightarrow \operatorname{Mod}(X)$ the embedding functor.

The support of $Y$ is the quasi-scheme

$$
\left(\operatorname{Mod}\left(o_{X} / o_{X}(-Y)\right), i^{*}\left(\mathcal{O}_{X}\right)\right),
$$

which we denote by

$$
\left(\operatorname{Mod}(Y), \mathcal{O}_{Y}\right)=\left(Y, \mathcal{O}_{Y}\right)
$$

Note that the letter " $Y$ " has a double role: On one hand, it denotes an effective divisor on $X$ (i.e. an invertible subobject of $o_{X}$ ). On the other hand, it denotes a quasi-scheme (namely the Grothendieck category $Y=\operatorname{Mod}(Y)$ ).

By proposition 1.7, part $1^{\circ}$, the quasi-scheme $\left(Y, \mathcal{O}_{Y}\right)$ is a bi-closed sub-quasischeme of $\left(X, \mathcal{O}_{X}\right)$. We shall denote the inclusion of $Y$ into $X$ by $i: Y \hookrightarrow X$; by the definition $Y=\operatorname{Mod}(Y)=\operatorname{Mod}\left(o_{X} / o_{X}(-Y)\right)$, this is consistent with the way $i_{*}$ was used above. Note that $i^{*}\left(\mathcal{O}_{X}\right)=\mathcal{O}_{Y}$.

By proposition 1.7 part $2^{\circ}$, if $\left(X, \mathcal{O}_{X}\right)$ is noetherian, then $\left(Y, \mathcal{O}_{Y}\right)$ is also noetherian.

To illustrate the concepts of the theory, let us look at an example.

Example 1.10 (The cubic divisor in the elliptic quantum plane). For this example, let $k$ be algebraically closed. Let $Y$ be a smooth curve of degree three in $\mathbb{P}_{k}^{2}$. The curve $Y$ is elliptic, and we let $\tau$ be a translation of $Y$. Denoting the inclusion by $i: Y \hookrightarrow \mathbb{P}^{2}$, we set $\mathcal{L}=i^{*} \mathcal{O}_{\mathbb{P}^{2}}(1)$.

By the methods of [1] we can associate to the data $(Y, \tau, \mathcal{L})$ a certain algebra, $A$, which has the following properties:

- It is connected graded Artin-Schelter regular with $\operatorname{gldim}(A)=3$.

- It has Hilbert series $H_{A}(t)=(1-t)^{-3}$, and consequently, $\operatorname{dim} A_{n}=\frac{1}{2} n^{2}+$ $\frac{3}{2} n+1$ for any $n \geq 0$.

- It contains a regular central element $g \in A_{3}$ such that $A /(g)$ is a so-called twisted homogeneous coordinate ring of $Y$. Moreover, we have $\left(Y, \mathcal{O}_{Y}\right)=$ $\operatorname{Proj}(A /(g))$.

We know that $\left(S, \mathcal{O}_{S}\right):=\operatorname{Proj}(A)=(\operatorname{Tails}(A), \pi A)$ is a quasi-scheme over $k$, known in the literature as "an elliptic quantum plane", and as we shall see, $Y$ is isomorphic to an effective divisor $T$ on $S$. We prove this in two steps. 
$1^{\circ}$ : There is an effective divisor $T$ on $S$, defined as follows: Consider the invertible bi-module $o_{S}(-T)$ on $S$ given by the functor $\mathcal{M} \longmapsto \mathcal{M}(3)$. There is a natural transformation of functors

$$
\lambda: \mathrm{id} \longrightarrow \mathcal{H} \text { om }\left(o_{S}(-T),-\right)=(-)(3)
$$

induced by multiplication with the regular central element $g$. This gives a morphism of bi-modules $o_{S}(-T) \longrightarrow o_{S}$, and we claim that the morphism is monic, and so determines an effective divisor $T$ on $S$.

To see monicness, observe that by [10 proof of 3.1.1(3)] it just means that $\lambda_{\mathcal{I}}$ is epic for each injective $\mathcal{I} \in \operatorname{Mod}(S)$. But $\lambda$ is induced by multiplication with $g$, so $\lambda_{\mathcal{I}}$ is obtained by taking a graded module $I$ with $\pi(I)=\mathcal{I}$ and setting $\lambda_{\mathcal{I}}=\pi(g \cdot)$. (We use $\pi$ in the sense of [2, p. 234] for the canonical functor $\operatorname{GrMod}(A) \longrightarrow \operatorname{Tails}(A)$.) By [2, prop. 7.1(1)], we can suppose that $I$ is injective in $\operatorname{GrMod}(A)$, and then $I \stackrel{g \cdot}{\longrightarrow} I(3)$ is surjective because $I$ is divisible. Since $\pi$ is exact, $\lambda_{\mathcal{I}}=\pi\left(g^{*}\right)$ is then epic as claimed.

$2^{\circ}: T$ can be identified with the curve $Y$ considered above. For this, note that proposition 1.7. part $4^{\circ}$, says that for $\mathcal{M} \in \operatorname{Mod}(S)$, we have $\mathcal{M} \in \operatorname{Mod}(T)$ precisely if $\lambda_{\mathcal{M}}=0$. That is, $\mathcal{M}$ is in $\operatorname{Mod}(T)$ precisely if $\mathcal{M}$ comes from a module annihilated by $g$. So

$$
\left(T, \mathcal{O}_{T}\right)=\operatorname{Proj}(A /(g))=\left(Y, \mathcal{O}_{Y}\right),
$$

where the latter "=" is one of the general properties given above.

As one of our basic tools, we need $K$-theory for quasi-schemes. In particular, we need filtered $K$-groups with filtration induced by Krull dimension.

Definition 1.11. Let $\left(X, \mathcal{O}_{X}\right)$ be a noetherian quasi-scheme over $k$. We can then consider Krull dimension of objects in $\bmod (X)$ (although not all objects may have Krull dimension). If $d$ is an integer, then we say that $X$ is $d$-dimensional if

- Each object $M \in \bmod (X)$ has a Krull dimension, and $\operatorname{Kdim}(M) \leq d$.

- There exists an object $M \in \bmod (X)$ with $\operatorname{Kdim}(M)=d$.

Note that if $\left(X, \mathcal{O}_{X}\right)$ is a noetherian quasi-scheme over $k$, and $Y$ is an effective divisor on $X$, and we consider an object $N \in \operatorname{Mod}(Y)$, then $N$ has the same subobjects whether we think of $N$ as sitting in $\operatorname{Mod}(Y)$ or in $\operatorname{Mod}(X)$, because $\operatorname{Mod}(Y)$ is bi-closed in $\operatorname{Mod}(X)$. Consequently, the Krull dimension of $N$ is also the same whether we compute it in $\bmod (X)$ or in $\bmod (Y)$.

Definition 1.12. Let $\left(X, \mathcal{O}_{X}\right)$ be a noetherian quasi-scheme over $k$. We write

$$
K(X)=K(\bmod (X)) .
$$

So $K(X)$ is the Grothendieck group of the abelian category $\bmod (X)$. If $M \in$ $\bmod (X)$, then we write $[M]$ for the class in $K(X)$ corresponding to $M$.

Definition 1.13. Let $\left(X, \mathcal{O}_{X}\right)$ be a noetherian quasi-scheme over $k$. For any integer $j \geq-1$, we set

$F_{j} K(X)=$ the subgroup of $K(X)$ generated by all $[M]$ 's with $\operatorname{Kdim}(M) \leq j$.

We clearly get a filtration on $K(X)$,

$$
0=F_{-1} K(X) \subseteq F_{0} K(X) \subseteq \cdots \subseteq F_{n} K(X) \subseteq \cdots
$$


which may not exhaust all of $K(X)$. But of course, in the case of a $d$-dimensional $X$, the filtration is finite and exhaustive, and of the form

$$
0=F_{-1} K(X) \subseteq F_{0} K(X) \subseteq \cdots \subseteq F_{d-1} K(X) \subseteq F_{d} K(X)=K(X) .
$$

If $\left(X, \mathcal{O}_{X}\right)$ is a noetherian quasi-scheme over $k$, and $Y$ is an effective divisor on $X$, then the inclusion

$$
i_{*}: \bmod (Y) \hookrightarrow \bmod (X)
$$

is an exact functor, and hence induces a group homomorphism

$$
K(i): K(Y) \longrightarrow K(X) .
$$

Moreover, since Krull dimension of a $\bmod (Y)$-object is the same whether we compute it in $\bmod (X)$ or in $\bmod (Y)$, the homomorphism $K(i)$ respects the filtration we have introduced on the $K$-groups. That is, for each integer $j$,

$$
K(i)\left(F_{j} K(Y)\right) \subseteq F_{j} K(X) .
$$

Having introduced $K$-theory, we go on to look at some conditions that we might want to impose if we have a noetherian quasi-scheme $\left(X, \mathcal{O}_{X}\right)$ over $k$, and possibly an effective divisor $Y$ on $X$. We will refer to these conditions throughout the manuscript, so for handy reference, they are collected in the following definition in one long list. The conditions are all rather natural: Some of them (such as [Euler] and [Dual]) tell us that $X$ behaves like a projective scheme, while others (such as $[(d-1)$-dim] and [Fixd comp] $)$ tell us that $Y$ sits in $X$ like a hypersurface.

Definition 1.14. In the following conditions, $\left(X, \mathcal{O}_{X}\right)$ denotes a noetherian quasischeme over $k$, and $Y$ denotes an effective divisor on $X$, and $d \geq 0$ denotes an integer which we sometimes think of as $X$ 's dimension.

[(d-1)-dim]: The noetherian quasi-scheme $Y$ is $(d-1)$-dimensional.

[Admissible]: We have $i^{!}\left(\mathcal{O}_{X}\right)=0$, where $i: Y \hookrightarrow X$ denotes the inclusion. (This is sometimes known as admissibility of the divisor $Y$ on $X$.)

[Fixd comp]: When $M \in \bmod (Y)$ has $\operatorname{Kdim}(M)=d-1$, we have $[M]-$ $[M(-Y)] \in F_{d-2} K(Y)$.

[Commutative]: The quasi-scheme $\left(Y, \mathcal{O}_{Y}\right)$ over $k$ is a commutative scheme.

[Euler]: If $M \in \bmod (X)$, then

$$
\begin{array}{ll}
\operatorname{dim}_{k} \operatorname{Ext}_{X}^{j}\left(\mathcal{O}_{X}, M\right)<\infty & \text { for any } j, \\
\operatorname{Ext}_{X}^{j}\left(\mathcal{O}_{X}, M\right)=0 & \text { for } j \gg 0 .
\end{array}
$$

So Euler characteristic is well-defined on $\bmod (X)$ by

$$
\chi_{X}(-)=\sum_{j}(-1)^{j} \operatorname{dim}_{k} \operatorname{Ext}_{X}^{j}\left(\mathcal{O}_{X},-\right) .
$$

[Invariant]: (This condition is only imposed when $\left(X, \mathcal{O}_{X}\right)$ satisfies condition [Euler].) If $p$ is a point on $X$, then $\chi_{X}(p(Y))=\chi_{X}(p)$. By a point on $X$, we mean a simple object of the category $\bmod (X)$. Note that when $p$ is a point, then the twist $p(Y)$ is also a point.

[Dual]: There is a dualizing object $\omega \in \bmod (X)$, characterized by the existence of isomorphisms for all integers $j$,

$$
\operatorname{Ext}_{X}^{j}\left(\mathcal{O}_{X}, M\right)^{\prime} \cong \operatorname{Ext}_{X}^{d-j}(M, \omega),
$$

natural in $M \in \bmod (X)$. The prime denotes dualization with respect to $k$. 
$[$ Dual $+\mathbf{K}]:\left(X, \mathcal{O}_{X}\right)$ satisfies condition [Dual], and the dualizing object $\omega$ satisfies

$$
[\omega]-\left[\mathcal{O}_{X}\right] \in F_{d-1} K(X) .
$$

One central point about definition 1.14 is that some of its conditions on $\left(X, \mathcal{O}_{X}\right)$ (such as [Euler] and [Dual]) are satisfied when $X$ is a good non-commutative projective scheme; see [2] and [12]. (Non-commutative projective schemes are really our main examples of quasi-schemes.) Another point is that if $\left(Y, \mathcal{O}_{Y}\right)$ is a good commutative scheme (the main example being a smooth projective curve), some of the definition's conditions on $Y$ are in fact satisfied. The proof of the following result is a matter of using well-known properties of commutative (projective) schemes.

Proposition 1.15. Suppose that $k$ is algebraically closed, let $\left(X, \mathcal{O}_{X}\right)$ be a noetherian quasi-scheme over $k$, and let $Y$ be an effective divisor on $X$. Suppose that $Y$ satisfies definition 1.14's condition [Commutative] and is of finite type, reduced, and irreducible.

Then $Y$ satisfies definition 1.14's condition [Fixd comp].

Let us round off with a result on Euler characteristic.

Lemma 1.16. Let $\left(X, \mathcal{O}_{X}\right)$ be a quasi-scheme over $k$, let $Y$ be an effective divisor on $X$, and let $i: Y \hookrightarrow X$ be the embedding.

$\left.1^{\circ}\right)$ Let $M \in \operatorname{Mod}(X)$ and $N \in \operatorname{Mod}(Y)$, and suppose that $i^{!}(M)=0$. Then there are isomorphisms

$$
\operatorname{Ext}_{X}^{j}(M, N) \cong \operatorname{Ext}_{Y}^{j}\left(i^{*}(M), N\right)
$$

for all integers $j$, natural in $M$ and $N$.

$\left.2^{\circ}\right)$ Suppose that $\left(X, \mathcal{O}_{X}\right)$ and $Y$ satisfy definition 1.14's conditions [Euler] and [Admissible], and let $N \in \bmod (Y)$. Then the canonical formula for Euler characteristic on $Y$,

$$
\chi_{Y}(N)=\sum_{j}(-1)^{j} \operatorname{dim}_{k} \operatorname{Ext}_{Y}^{j}\left(\mathcal{O}_{Y}, N\right)
$$

makes sense, and we have

$$
\chi_{Y}(N)=\chi_{X}(N) .
$$

Proof. $1^{\circ}$ : See [10, prop. 5.1.2(1)].

$2^{\circ}$ : This clearly follows from $1^{\circ}$, which gives

$$
\operatorname{dim}_{k} \operatorname{Ext}_{Y}^{j}\left(\mathcal{O}_{Y}, N\right)=\operatorname{dim}_{k} \operatorname{Ext}_{Y}^{j}\left(i^{*} \mathcal{O}_{X}, N\right)=\operatorname{dim}_{k} \operatorname{Ext}_{X}^{j}\left(\mathcal{O}_{X}, N\right)
$$

for each integer $j$, because we have $i^{!} \mathcal{O}_{X}=0$ by condition [Admissible].

\section{Positive multiples of EFFective Divisors}

It will turn out to be useful that one can define positive multiples of an effective divisor; in particular when one combines positive multiples with the Riemann-Roch theorem to be proved in section 5 The present section, which is purely technical, defines such positive multiples, and shows in propositions 2.4 to 2.6 that some of the important properties from definition 1.14 are inherited from an effective divisor to its positive multiples. These results are summed up in remark 2.7] Finally, a result on the connection with $K$-theory is shown in lemma 2.8 . 
Definition 2.1. Let $\left(X, \mathcal{O}_{X}\right)$ be a quasi-scheme over $k$, and let $Y$ be an effective divisor on $X$ defined by $o_{X}(-Y) \hookrightarrow o_{X}$ corresponding to the natural transformation

$$
\lambda: \text { id } \longrightarrow \mathcal{H o m}_{X}\left(o_{X}(-Y),-\right) .
$$

Recall from definition 1.4 that we have $o_{X}(-n Y)=o_{X}(-Y)^{\otimes n}$ for any integer $n$. Now let $n \geq 0$. We construct morphisms $o_{X}(-n Y) \longrightarrow o_{X}$ inductively, as follows:

First we set $\lambda_{0}$ equal to the identity on $\mathcal{H o m}\left(o_{X},-\right)$. This defines a morphism $o_{X}(0 Y) \longrightarrow o_{X}$, since $o_{X}(0 Y)=o_{X}$.

Next, suppose that the morphism $o_{X}(-(n-1) Y) \longrightarrow o_{X}$ is given by the natural transformation $\lambda_{n-1}:$ id $\longrightarrow \mathcal{H} m_{X}\left(o_{X}(-(n-1) Y),-\right)$. We then let $o_{X}(-n Y) \longrightarrow$ $o_{X}$ be given by the natural transformation $\lambda_{n}$ defined by the composition

$$
\begin{gathered}
\mathcal{H} o m\left(o_{X}(-Y),-\right) \\
\mathcal{H o m}\left(o_{X}(-Y), \lambda_{n-1}\right) \\
\mathcal{H o m}\left(o_{X}(-Y), \mathcal{H o m}\left(o_{X}(-(n-1) Y),-\right)\right)=\mathcal{H} o m\left(o_{X}(-n Y),-\right) .
\end{gathered}
$$

Note that of course, $\lambda_{1}=\lambda$.

Like $o_{X}(-n Y)$, the morphism $\lambda_{n}$ is only defined up to unique isomorphism.

The rest of the manuscript uses the notation set up in definition 2.1 (including the $\lambda_{i}$ 's) without comments.

Note that the construction of the morphism $o_{X}(-n Y) \longrightarrow o_{X}$ could also be written as follows: Suppose given $o_{X}(-(n-1) Y) \longrightarrow o_{X}$. Tensor it with $o_{X}(-Y)$ to get

$$
o_{X}(-Y) \otimes o_{X}(-(n-1) Y) \longrightarrow o_{X}(-Y) \otimes o_{X}=o_{X}(-Y),
$$

and compose this morphism with the inclusion $o_{X}(-Y) \longrightarrow o_{X}$. This also makes it easy to see inductively that each $o_{X}(-n Y) \longrightarrow o_{X}$ is monic.

Of course, as suggested by the notation, $o_{X}(-n Y)$ determines an effective divisor which we denote $n Y$, for any $n \geq 0$ :

Proposition 2.2. Let $\left(X, \mathcal{O}_{X}\right)$ be a quasi-scheme over $k$, let $Y$ be an effective divisor on $X$ defined by $o_{X}(-Y) \hookrightarrow o_{X}$, and let $n \geq 0$ be an integer.

Then the monic $o_{X}(-n Y) \hookrightarrow o_{X}$, as defined in definition 2.1, determines a well-defined effective divisor, $n Y$, on $X$.

Proof. First recall that an effective divisor is in fact defined as an invertible subobject of $o_{X}$. However, our monic $o_{X}(-n Y) \hookrightarrow o_{X}$ is determined up to unique isomorphism, so it determines a subobject of $o_{X}$ which is well-defined, that is, independent of the choice of $o_{X}(-Y) \hookrightarrow o_{X}$.

We have left to see that the bi-module $o_{X}(-n Y)$ is invertible, but that is obvious since $o_{X}(-n Y)$ is a tensor product of invertible bi-modules.

Note that we have not tried to attach any meaning to "the divisor $n Y$ for $n \leq$ -1 ". In other words, we will always stick with effective divisors, and will not try to define what the word "divisor" might mean on its own. 
Lemma 2.3. Let $\left(X, \mathcal{O}_{X}\right)$ be a noetherian quasi-scheme over $k$, let $Y$ be an effective divisor on $X$, and let $n \geq 1$ be an integer.

$\left.1^{\circ}\right)$ We have, as subcategories of $\operatorname{Mod}(X)$,

$$
\operatorname{Mod}(Y) \subseteq \operatorname{Mod}(n Y) .
$$

$\left.2^{\circ}\right)$ If $M \in \bmod (n Y)$ is $\operatorname{Kdim}$-critical (of any dimension), then $M \in \bmod (Y)$.

Proof. $1^{\circ}$ : Using the notation of definition 2.1, we obtain the natural transformation $\lambda_{n}$ which determines $o_{X}(-n Y) \hookrightarrow o_{X}$ as

$$
\lambda_{n}=\lambda_{n-1}(Y) \circ \lambda_{1}
$$

$\left(\lambda_{n-1}(Y)\right.$ denotes the shift of $\lambda_{n-1}$ by $\left.Y\right)$. And combining this with proposition 1.7. part $4^{\circ}$, we get the implications

$$
M \in \operatorname{Mod}(Y) \Leftrightarrow\left(\lambda_{1}\right)_{M}=0 \Rightarrow\left(\lambda_{n}\right)_{M}=0 \Leftrightarrow M \in \operatorname{Mod}(n Y) .
$$

$2^{\circ}$ : We use induction on $n$. So we suppose that any Kdim-critical object in $\bmod ((n-1) Y)$ is in $\bmod (Y)$.

Let $M \notin \bmod (Y)$ be $\ell$-critical with respect to Kdim. We aim to prove that $M \notin \bmod (n Y)$.

By proposition 1.7 part $4^{\circ}$, we know $\left(\lambda_{1}\right)_{M} \neq 0$. And by induction, we have $M \notin \bmod ((n-1) Y)$, hence $\left(\lambda_{n-1}\right)_{M} \neq 0$ by proposition 1.7 part $4^{\circ}$, hence

$$
\left(\lambda_{n-1}\right)_{M}(Y) \neq 0 \text {. }
$$

We now use equation (2.1), by which the map $\left(\lambda_{n}\right)_{M}$ is obtained as the composition

$$
M \stackrel{\left(\lambda_{1}\right)_{M}}{\longrightarrow} M(Y) \stackrel{\left(\lambda_{n-1}\right)_{M}(Y)}{\longrightarrow} M(n Y) .
$$

Since both maps here are non-zero while all three objects are $\ell$-critical it follows that both maps are monic, whence the composition of the maps is monic and, in particular, non-zero. So $M \notin \bmod (n Y)$ by proposition 1.7 part $4^{\circ}$.

Not only can we get new divisors $n Y$ from the divisor $Y$; it is also true that $n Y$ will inherit some good properties from $Y$.

Proposition 2.4. Let $\left(X, \mathcal{O}_{X}\right)$ be a noetherian quasi-scheme over $k$, let $Y$ be an effective divisor on $X$ satisfying definition 1.14's condition [(d-1)-dim], and let $n \geq 1$ be an integer. $\operatorname{dim}]$.

Then the effective divisor $n Y$ also satisfies definition 1.14]'s condition $[(d-1)$ -

Proof. We are given a $Y$ satisfying condition [( $d-1)$-dim], and use induction on $n$. So we suppose that $(n-1) Y$ satisfies condition [( $d-1)$-dim].

On one hand, we have $\operatorname{Mod}(Y) \subseteq \operatorname{Mod}(n Y)$ by lemma 2.3, and this makes it clear that $n Y$ is at least $(d-1)$-dimensional, since we have assumed that $Y$ is $(d-1)$-dimensional.

On the other hand, suppose that $M \in \bmod (X)$ has

$$
\operatorname{Kdim}(M) \not \leq d-1
$$

(note that this could mean that $M$ does not have Krull dimension at all). We want to see that $M \notin \bmod (n Y)$. Letting $i: Y \hookrightarrow X$ and $j:(n-1) Y \hookrightarrow X$ be the 
inclusions, we get short exact sequences from proposition 1.7, part $3^{\circ}$,

$$
\begin{gathered}
0 \rightarrow i^{!} M \longrightarrow M \stackrel{\left(\lambda_{1}\right)_{M}}{\longrightarrow} M(Y) \longrightarrow R^{1} i^{!} M \rightarrow 0, \\
0 \rightarrow j^{!} M \longrightarrow M \stackrel{\left(\lambda_{n-1}\right)_{M}}{\longrightarrow} M((n-1) Y) \longrightarrow R^{1} j^{!} M \rightarrow 0 .
\end{gathered}
$$

By induction, both $i^{!} M$ and $j^{!} M$ have Krull dimension less than or equal to $d-1$. The first sequence then implies

$$
\operatorname{Kdim}\left(\operatorname{Im}\left(\lambda_{1}\right)_{M}\right) \not \leq d-1,
$$

and the second sequence implies $\operatorname{Kdim}\left(\operatorname{Ker}\left(\lambda_{n-1}\right)_{M}\right) \leq d-1$, and hence

$$
\operatorname{Kdim}\left(\operatorname{Ker}\left(\lambda_{n-1}\right)_{M}(Y)\right) \leq d-1 .
$$

But this shows that

$$
\operatorname{Im}\left(\lambda_{1}\right)_{M} \not \subset \operatorname{Ker}\left(\lambda_{n-1}\right)_{M}(Y) .
$$

However, by equation (2.1), we then see that $\left(\lambda_{n}\right)_{M} \neq 0$, whence by proposition 1.7 part $4^{\circ}$, we have $M \notin \bmod (n Y)$.

Proposition 2.5. Let $\left(X, \mathcal{O}_{X}\right)$ be a noetherian quasi-scheme over $k$, let $Y$ be an effective divisor on $X$ satisfying definition 1.14's condition [Admissible], and let $n \geq 1$ be an integer.

Then the effective divisor $n Y$ also satisfies definition 1.14's condition [Admissible].

Proof. The proposition is trivial for $n=1$. For larger $n$ 's we induct on $n$, assuming that the effective divisor $(n-1) Y$ satisfies condition [Admissible].

Letting $i: Y \hookrightarrow X$ and $j:(n-1) Y \hookrightarrow X$ denote the inclusions, we have the sequences (2.2). The assumption of condition [Admissible] for $Y$ and $(n-1) Y$ says that $i^{!}\left(\mathcal{O}_{X}\right)=0$ and $j^{!}\left(\mathcal{O}_{X}\right)=0$, hence that $\left(\lambda_{1}\right)_{\mathcal{O}_{X}}$ and $\left(\lambda_{n-1}\right)_{\mathcal{O}_{X}}$ are monics. The latter fact means that $\left(\lambda_{n-1}\right)_{\mathcal{O}_{X}}(Y)$ is also monic, and hence by equation (2.1), we see that $\left(\lambda_{n}\right)_{\mathcal{O}_{X}}$ is monic.

But then if $\ell: n Y \hookrightarrow X$ denotes the inclusion, the sequence (1.2) used on the divisor $n Y$ implies that $\ell^{!}\left(\mathcal{O}_{X}\right)=0$, so $n Y$ satisfies condition [Admissible].

Proposition 2.6. Let $\left(X, \mathcal{O}_{X}\right)$ be a noetherian quasi-scheme over $k$, let $Y$ be an effective divisor on $X$ satisfying definition 1.14's condition [Fixd comp], and let $n \geq 1$ be an integer.

Then the effective divisor $n Y$ also satisfies definition [1.14's condition [Fixd comp].

Proof. We must show that if $M \in \bmod (n Y)$ is $(d-1)$-dimensional, then $[M]-$ $[M(-n Y)] \in F_{d-2} K(n Y)$.

By the existence of critical composition series, we can assume that $M$ is $(d-1)$ critical. But then $M \in \bmod (Y)$ by lemma 2.3] part $2^{\circ}$, so there is the equation $[M]-[M(-Y)] \in F_{d-2} K(Y)$ in $K$-theory for $Y$. Twisting yields

$$
[M(-m Y)]-[M(-(m+1) Y)] \in F_{d-2} K(Y)
$$

for each $m$, and adding these for $m=0, \ldots, n-1$ gives

$$
[M]-[M(-n Y)] \in F_{d-2} K(Y) .
$$

And this certainly implies $[M]-[M(-n Y)] \in F_{d-2} K(n Y)$. 
Remark 2.7. Note that as consequence of propositions [2.4, 2.5] and [2.6, if $\left(X, \mathcal{O}_{X}\right)$ is a noetherian quasi-scheme over $k$, and $Y$ is an effective divisor on $X$ such that $Y$ satisfies definition 1.14 s conditions [( $d-1)$-dim], [Admissible], and [Fixd comp], then each positive multiple $n Y$ satisfies the same three conditions.

Lemma 2.8. Let $\left(X, \mathcal{O}_{X}\right)$ be a noetherian quasi-scheme over $k$, and let $Y$ be an effective divisor on $X$ which satisfies definition 1.14's conditions [ $(d-1)$-dim], [Admissible], and [Fixd comp].

Then for each integer $n \geq 0$, we have that in $K(X)$,

$$
\left[\mathcal{O}_{n Y}\right]-n\left[\mathcal{O}_{Y}\right] \in F_{d-2} K(X) .
$$

Proof. For $n=0$ and $n=1$, the conclusion of the lemma is trivial. For larger $n$ 's, we induct on $n$, assuming that $(n-1) Y$ satisfies the conclusion of the lemma.

First note that since $Y$ satisfies condition [Admissible], so does each positive multiple $m Y$ by proposition 2.5. So using the exact sequence (1.4), we clearly have the equation in $K(X)$,

$$
\left[\mathcal{O}_{m Y}\right]=\left[\mathcal{O}_{X}\right]-\left[\mathcal{O}_{X}(-m Y)\right],
$$

and if one twists this equation by $-\ell Y$, one gets

$$
\left[\mathcal{O}_{m Y}(-\ell Y)\right]=\left[\mathcal{O}_{X}(-\ell Y)\right]-\left[\mathcal{O}_{X}(-(m+\ell) Y)\right] .
$$

This equation is valid for any integers $\ell, m$ with $m \geq 1$.

So in particular, let us write out what equation (2.3) says for some pairs of integers. For $\ell=0$ and $m=n$, we have

$$
\left[\mathcal{O}_{n Y}\right]=\left[\mathcal{O}_{X}\right]-\left[\mathcal{O}_{X}(-n Y)\right]
$$

for $\ell=0$ and $m=n-1$, we have

$$
\left[\mathcal{O}_{(n-1) Y}\right]=\left[\mathcal{O}_{X}\right]-\left[\mathcal{O}_{X}(-(n-1) Y)\right]
$$

and for $\ell=n-1$ and $m=1$, we have

$$
\left[\mathcal{O}_{Y}(-(n-1) Y)\right]=\left[\mathcal{O}_{X}(-(n-1) Y)\right]-\left[\mathcal{O}_{X}(-n Y)\right] .
$$

Subtracting the first of these equations from the sum of the latter two produces the result

$$
\left[\mathcal{O}_{n Y}\right]=\left[\mathcal{O}_{(n-1) Y}\right]+\left[\mathcal{O}_{Y}(-(n-1) Y)\right] .
$$

Next, condition [Fixd comp] is valid for $(n-1) Y$ by proposition 2.6, hence we have

$$
\left[\mathcal{O}_{Y}(-(n-1) Y)\right]-\left[\mathcal{O}_{Y}\right] \in F_{d-2} K(X) .
$$

Now we combine the equations: By induction,

$$
\left[\mathcal{O}_{(n-1) Y}\right]-(n-1)\left[\mathcal{O}_{Y}\right] \in F_{d-2} K(X) .
$$

Adding equations (2.5) and (2.6), we have

$$
\left[\mathcal{O}_{(n-1) Y}\right]+\left[\mathcal{O}_{Y}(-(n-1) Y)\right]-n\left[\mathcal{O}_{Y}\right] \in F_{d-2} K(X) .
$$

And because of equation (2.4), this reads

$$
\left[\mathcal{O}_{n Y}\right]-n\left[\mathcal{O}_{Y}\right] \in F_{d-2} K(X) .
$$




\section{INTERSECTING WITH AN EFFECTIVE DIVISOR}

This section defines the first Chern class $c(Y)$ of an effective divisor $Y$ (definition 3.1), and proves a few key properties, the most important one being lemma 3.4 which deals with the connection between $c(Y)$ and $K$-theory.

Definition 3.1. Let $\left(X, \mathcal{O}_{X}\right)$ be a noetherian quasi-scheme over $k$, and let

$$
\mathcal{M} \in \operatorname{BIMOD}(X, X)
$$

be an invertible bi-module. Clearly

$$
[M] \longmapsto[M] \otimes \mathcal{M}^{-1}:=\left[M \otimes \mathcal{M}^{-1}\right]
$$

is a well-defined automorphism of $K(X)$, and we use it to define an endomorphism $c(\mathcal{M})$ of $K(X)$, called "the first Chern class of $\mathcal{M}$ ", by

$$
c(\mathcal{M})([M])=[M]-[M] \otimes \mathcal{M}^{-1} .
$$

In particular, let $Y$ be an effective divisor on $X$. We define

$$
c(Y)=c\left(o_{X}(Y)\right) \text {; }
$$

that is,

$$
c(Y)([M])=[M]-[M(-Y)] .
$$

This definition can already be made in commutative geometry. Moreover, in the commutative setting, for any integer $i$ it is true that

$$
c(Y)\left(F_{i} K(X)\right) \subseteq F_{i-1} K(X),
$$

and hence, one gets well-defined maps

$$
F_{i} K(X) / F_{i-1} K(X) \longrightarrow F_{i-1} K(X) / F_{i-2} K(X) .
$$

Defining the $i$ th Chow group of $X$ as $A_{i}(X)=F_{i} K(X) / F_{i-1} K(X)$ (cf. 3, ex. 1.6.5]), we therefore see that $c(Y)$ defines intersection operators between Chow groups,

$$
A_{i}(X) \stackrel{Y \cap(-)}{\longrightarrow} A_{i-1}(X) .
$$

This approach fails in the non-commutative case, because equation (3.1) is not true in general (although something does hold; see lemma 3.4). We therefore refrain from defining Chow groups, and stick with the $K$-groups themselves. And here our philosophy will be the following: If $[M]$ represents a "closed subscheme" of $X$ (e.g. $M$ could be a quotient of $\mathcal{O}_{X}$ ), one should think of the $K$-class $c(Y)([M])$ as representing the intersection of the divisor $Y$ with the closed subscheme given by $[M]$.

Lemma 3.2. Let $\left(X, \mathcal{O}_{X}\right)$ be a noetherian quasi-scheme over $k$, and let $Y$ be an effective divisor on $X$. Then for any $M \in \bmod (X)$, we have the equation in $K(X)$,

$$
c(Y)([M])=\left[i^{*} M\right]-\left[\left(i^{!} M\right)(-Y)\right] .
$$

Proof. This follows immediately from the exact sequence (1.4), which gives the second "=" in the following computation:

$$
c(Y)([M])=[M]-[M(-Y)]=\left[i^{*} M\right]-\left[\left(i^{!} M\right)(-Y)\right] .
$$


Example 3.3 (Intersecting with the whole scheme). Let $\left(X, \mathcal{O}_{X}\right)$ be a noetherian quasi-scheme over $k$, and let $Y$ be an effective divisor on $X$ which satisfies condition [Admissible]. Then

$$
c(Y)\left(\left[\mathcal{O}_{X}\right]\right)=\left[i^{*} \mathcal{O}_{X}\right]-\left[\left(i^{!} \mathcal{O}_{X}\right)(-Y)\right]=\left[i^{*} \mathcal{O}_{X}\right]=\left[\mathcal{O}_{Y}\right],
$$

where the first "=" follows from lemma 3.2. In other words, intersecting $Y$ with "everything" gives us the structure sheaf of $Y$, as one would expect.

Lemma 3.4. Let $\left(X, \mathcal{O}_{X}\right)$ be a noetherian quasi-scheme over $k$, and let $Y$ be an effective divisor on $X$. Then

$\left.1^{\circ}\right)$ The endomorphism $c(Y)$ maps $K(X)$ into the image of the canonical map

$$
K(Y) \longrightarrow K(X) \text {. }
$$

$\left.2^{\circ}\right)$ If $Y$ satisfies condition [Fixd comp], then the endomorphism $c(Y)$ maps $F_{d-1} K(X)$ into $F_{d-2} K(X)$.

Proof. $1^{\circ}$ : From lemma 3.2 we have

$$
c(Y)([M])=\left[i^{*} M\right]-\left[\left(i^{!} M\right)(-Y)\right]
$$

in $K(X)$. But $i^{*} M$ and $i^{!} M$ are in $\bmod (Y)$, and using proposition 1.7 part $5^{\circ}$, we see that so is $\left(i^{!} M\right)(-Y)$. So $c(Y)([M])$ is in the image of the canonical map $K(Y) \longrightarrow K(X)$.

$2^{\circ}:$ It is clear from the definition of $c(Y)$ that it maps $F_{d-2} K(X)$ to $F_{d-2} K(X)$. Thus, all we need to see is that when $M$ is $(d-1)$-dimensional, we have $c(Y)([M]) \in$ $F_{d-2} K(X)$. And this is immediate from condition [Fixd comp].

Lemma 3.5. Let $\left(X, \mathcal{O}_{X}\right)$ be a noetherian quasi-scheme over $k$, and let $\mathcal{M}, \mathcal{N} \in$ $\operatorname{BIMOD}(X, X)$ be two invertible bi-modules. Then as endomorphisms of $K(X)$, we have

$$
c(\mathcal{M} \otimes \mathcal{N})=(c(\mathcal{M})+c(\mathcal{N}))-c(\mathcal{M}) \circ c(\mathcal{N}) .
$$

Proof. Let $M \in \bmod (X)$. We can compute as follows:

$$
\begin{aligned}
&(c(\mathcal{M})+c(\mathcal{N}))([M])-c(\mathcal{M} \otimes \mathcal{N})([M]) \\
&=\left([M]-[M] \otimes \mathcal{M}^{-1}+[M]-[M] \otimes \mathcal{N}^{-1}\right) \\
&-\left([M]-[M] \otimes \mathcal{N}^{-1} \otimes \mathcal{M}^{-1}\right) \\
&= {[M]-[M] \otimes \mathcal{N}^{-1}-[M] \otimes \mathcal{M}^{-1}+[M] \otimes \mathcal{N}^{-1} \otimes \mathcal{M}^{-1} } \\
&=\left([M]-[M] \otimes \mathcal{N}^{-1}\right)-\left([M]-[M] \otimes \mathcal{N}^{-1}\right) \otimes \mathcal{M}^{-1} \\
&= c(\mathcal{M}) \circ c(\mathcal{N})([M]) .
\end{aligned}
$$

\section{InterseCtion MULTiPLiCities ON SURFACES}

This section uses the Chern class $c(Y)$ to introduce the intersection multiplicity mapping $\langle Y,-\rangle$. Under suitable conditions, it then proves some crucial properties of $\langle Y,-\rangle$ : A self intersection formula (theorem 4.5) and some additivity results (theorem 4.8 to corollary 4.11).

Definition 4.1. Let $\left(X, \mathcal{O}_{X}\right)$ be a noetherian quasi-scheme over $k$, satisfying condition [Euler], and let $Y$ be an effective divisor on $X$. We define a homomorphism

$$
\langle Y,-\rangle: K(X) \longrightarrow \mathbb{Z},\langle Y, z\rangle=\chi(c(Y)(z)) .
$$


Most of the time, we will in fact think of $\langle Y,-\rangle$ as being defined just on $F_{1} K(X)$. It is, however, handy to allow $\langle Y,-\rangle$ to be defined on all of $K(X)$. If $C \in \bmod (X)$ is 1-dimensional (i.e. a curve module), then we think of the integer $\langle Y,[C]\rangle$ as "the intersection multiplicity of the hypersurface $Y$ with the curve $C$ ".

Before going on to prove properties of $\langle Y,-\rangle$, let us remark that our intersection multiplicity is closely connected with the intersection multiplicity introduced in 7 def. 8.8]. In [7, the bi-linear form on $K(X)$ defined by

$$
([M],[N])_{X}=\sum_{j}(-1)^{j} \operatorname{dim}_{k} \operatorname{Ext}_{X}^{j}(M, N)
$$

is introduced, and used to define the intersection multiplicity

$$
[M] \cdot[N]=-([M],[N])_{X} .
$$

Of course, for this to work, one has to require that $X$ satisfies that for any $M, N \in$ $\bmod (X)$, we have

$$
\begin{array}{ll}
\operatorname{dim}_{k} \operatorname{Ext}_{X}^{j}(M, N)<\infty & \text { for any } j, \\
\operatorname{Ext}_{X}^{j}(M, N)=0 & \text { for } j \gg 0 .
\end{array}
$$

The connection between our intersection multiplicity and the one defined in [7] is now given by

Proposition 4.2. Let $\left(X, \mathcal{O}_{X}\right)$ be a noetherian quasi-scheme over $k$, satisfying conditions (4.1), and let $Y$ be an effective divisor on $X$ satisfying [Admissible]. Then $\left(X, \mathcal{O}_{X}\right)$ satisfies [Euler], and if $z \in K(X)$, then

$$
\langle Y, z\rangle=\left[\mathcal{O}_{Y}\right] \cdot z(-Y) .
$$

Proof. It is clear that $\left(X, \mathcal{O}_{X}\right)$ satisfies [Euler], and the equation can be checked by direct computation:

$$
\begin{aligned}
\langle Y, z\rangle & =\chi(c(Y)(z)) \\
& =\chi(z-z(-Y)) \\
& =\chi(z)-\chi(z(-Y)) \\
& =\left(\left[\mathcal{O}_{X}\right], z\right)_{X}-\left(\left[\mathcal{O}_{X}\right], z(-Y)\right)_{X} \\
& =\left(\left[\mathcal{O}_{X}\right], z\right)_{X}-\left(\left[\mathcal{O}_{X}(Y)\right], z\right)_{X} \\
& =\left(\left[\mathcal{O}_{X}\right]-\left[\mathcal{O}_{X}(Y)\right], z\right)_{X} \\
& \stackrel{(a)}{=}\left(-\left[i^{*}\left(\mathcal{O}_{X}\right)(Y)\right], z\right)_{X} \\
& =-\left(\left[\mathcal{O}_{Y}(Y)\right], z\right)_{X} \\
& =-\left(\left[\mathcal{O}_{Y}\right], z(-Y)\right)_{X} \\
& =\left[\mathcal{O}_{Y}\right] \cdot z(-Y),
\end{aligned}
$$

where " $(a)$ " is by the sequence (1.4), and condition [Admissible] for $Y$.

The connection between the two intersection forms $\langle Y,-\rangle$ and $\left[\mathcal{O}_{Y}\right] \cdot(-)$ is often even closer than indicated by the proposition: If one restricts to $z \in F_{1} K(X)$, it will frequently be true that $z-z(-Y) \in F_{0} K(X)$, and that $\left[\mathcal{O}_{Y}\right] \cdot(-)$ vanishes on $F_{0} K(X)$. In this case, proposition 4.2 can be improved to

$$
\langle Y, z\rangle=\left[\mathcal{O}_{Y}\right] \cdot z(-Y)=\left[\mathcal{O}_{Y}\right] \cdot z .
$$

In the following simple example, $\langle Y,-\rangle$ behaves as one would expect. 
Example 4.3 (Intersecting with a curve in good position). Let $\left(X, \mathcal{O}_{X}\right)$ be a noetherian quasi-scheme over $k$, and let $Y$ be an effective divisor on $X$ which satisfies condition [Commutative] in a way such that $\left(Y, \mathcal{O}_{Y}\right)$ is of finite type.

We now borrow some terminology from [9]: We let $C \in \bmod (X)$ be a pure curve module, that is, we have $\operatorname{Kdim}(C)=1$ and $i^{!}(C)=0$. We also suppose that $i^{*}(C)$ is semisimple, and write

$$
i^{*}(C)=p_{1} \oplus \cdots \oplus p_{n}
$$

for some points $p_{1}, \ldots, p_{n}$ on $Y$. (Recall that a point on $X$ is just a simple object of $\bmod (X)$; when $p$ is a point on $Y$ in the usual sense, we identify it with the skyscraper sheaf $\mathcal{O}_{p}$ at $p$, which is indeed a simple object of $\bmod (Y)$, hence a simple object of $\bmod (X)$.) Note that condition (4.2) is among the conditions in $[9]$ which characterize curve modules in good position with respect to $Y$. 9] thinks of the points $p_{1}, \ldots, p_{n}$ as the intersection of $Y$ and $C$, and of the number $n$ as the intersection multiplicity between $Y$ and $C$.

We then have

$$
c(Y)([C])=\left[i^{*}(C)\right]=\left[p_{1} \oplus \cdots \oplus p_{n}\right]=\left[p_{1}\right]+\cdots+\left[p_{n}\right],
$$

where the first "=" follows from lemma 3.2 So $c(Y)$ gives the correct intersection between $Y$ and $C$. If moreover all points $p_{i}$ have Euler characteristic 1, then we also get

$$
\langle Y,[C]\rangle=\chi(c(Y)([C]))=\chi\left(\left[p_{1}\right]+\cdots+\left[p_{n}\right]\right)=n,
$$

the correct intersection number. The condition that all points have Euler characteristic one is satisfied for instance if $k$ is algebraically closed.

From now on, the integer $d$ mentioned in definition 1.14 will always be equal to 2 . We therefore also write "condition [1-dim]" rather than "condition [(d-1)-dim]" (cf. definition 1.14).

Now consider a situation where we have a noetherian quasi-scheme $\left(X, \mathcal{O}_{X}\right)$ over $k$, and an effective divisor $Y$ on $X$ satisfying [Commutative] in a way such that $\left(Y, \mathcal{O}_{Y}\right)$ is a smooth projective curve. In [10, sec. 3.7], the normal bundle of $Y$ in $X$ is defined as the bi-module

$$
\mathcal{N}_{Y / X}=o_{X}(Y) / o_{X},
$$

and in [10, sec. 5.1] it is remarked that there is a line bundle (in the usual commutative sense) $\mathcal{N}$ on $Y$, and an automorphism (in the usual commutative sense) $\tau$ of $Y$, such that

$$
\left(-\otimes \mathcal{N}_{Y / X}\right)=\tau_{*}\left(-\otimes_{\mathcal{O}_{Y}} \mathcal{N}\right)
$$

as functors on $\operatorname{Mod}(Y)$. It is understood that in the formula, the right hand side acts on objects of $\operatorname{Mod}(Y)$ by means of the chosen identification of $Y$ with a commutative curve.

Theorem 4.5 shows that in this setup our definitions have further good properties, in that the classical self intersection formula, $\left\langle Y,\left[\mathcal{O}_{Y}\right]\right\rangle=\operatorname{deg}(\mathcal{N})$, is valid. Before embarking on the theorem we need a small lemma.

Lemma 4.4. Let $\left(X, \mathcal{O}_{X}\right)$ be a noetherian quasi-scheme over $k$, and let $Y$ be an effective divisor on $X$, satisfying [Commutative] in a way such that $\left(Y, \mathcal{O}_{Y}\right)$ is a smooth projective curve. Let $\mathcal{N}_{Y / X}=o_{X}(Y) / o_{X}$ be the normal bundle of $Y$ in $X$ as defined in [10, sec. 3.7] and above, and let $\tau$ and $\mathcal{N}$ be determined in terms of $\mathcal{N}_{Y / X}$ as described above. 
Then for $M \in \operatorname{Mod}(Y)$, there are natural isomorphisms,

$$
\begin{gathered}
M(Y)=M \otimes \mathcal{N}_{Y / X}=\tau_{*}\left(M \otimes_{\mathcal{O}_{Y}} \mathcal{N}\right), \\
M(-Y)=\mathcal{H} \operatorname{om}\left(\mathcal{N}_{Y / X}, M\right)=\tau_{*}^{-1}(M) \otimes_{\mathcal{O}_{Y}} \mathcal{N}^{-1} .
\end{gathered}
$$

Proof. The short exact sequence

$$
0 \rightarrow o_{X}(-Y) \longrightarrow o_{X} \longrightarrow o_{Y} \rightarrow 0
$$

gives rise to the short exact sequence

$$
0 \rightarrow o_{X} \longrightarrow o_{X}(Y) \longrightarrow \mathcal{N}_{Y / X} \rightarrow 0 .
$$

Let $M \in \operatorname{Mod}(Y)$. Then we know that $M \stackrel{\lambda_{M}}{\longrightarrow} M(Y)$ is zero. Hence the leftmost morphism is zero in the right exact sequence

$$
M \otimes o_{X} \longrightarrow M \otimes o_{X}(Y) \longrightarrow M \otimes \mathcal{N}_{Y / X} \rightarrow 0,
$$

yielding

$$
M(Y)=M \otimes \mathcal{N}_{Y / X}
$$

and since we already know that

$$
M \otimes \mathcal{N}_{Y / X}=\tau_{*}\left(M \otimes_{\mathcal{O}_{Y}} \mathcal{N}\right),
$$

we have proved equation (4.3).

An entirely similar computation yields that for $M \in \operatorname{Mod}(Y)$ we have

$$
M(-Y)=\mathcal{H o m}\left(\mathcal{N}_{Y / X}, M\right) .
$$

Now, $\operatorname{Mod}(Y)$ is stable under the inverse equivalences $M \longmapsto M(Y)$ and $M \longmapsto$ $M(-Y)$ by proposition 1.7, part $5^{\circ}$. So if we think of $M \longmapsto M(Y)$ as giving an autoequivalence of $\operatorname{Mod}(Y)$, its inverse is $M \longmapsto M(-Y)$. However, equation (4.3) allows us to compute the inverse of $M \longmapsto M(Y)$ differently, as

$$
M \longmapsto \tau_{*}^{-1}(M) \otimes_{\mathcal{O}_{Y}} \mathcal{N}^{-1},
$$

and so the two inverses must be equivalent, that is, for $M \in \operatorname{Mod}(Y)$ we have

$$
M(-Y)=\tau_{*}^{-1}(M) \otimes_{\mathcal{O}_{Y}} \mathcal{N}^{-1} .
$$

Combining equations (4.5) and (4.6), we get equation (4.4).

Theorem 4.5 (The self intersection theorem). Let $\left(X, \mathcal{O}_{X}\right)$ be a noetherian quasischeme over an algebraically closed field $k$, satisfying condition [Euler], and let $Y$ be an effective divisor on $X$ satisfying condition [Commutative], such that $\left(Y, \mathcal{O}_{Y}\right)$ is a smooth projective curve.

Let $\mathcal{N}_{Y / X}$ be $Y$ 's normal bundle in $X$, as defined in [10, sec. 3.7], and let the line bundle $\mathcal{N}$ be determined in terms of $\mathcal{N}_{Y / X}$ as described before lemma 4.4. We now have the "self intersection formula",

$$
\left\langle Y,\left[\mathcal{O}_{Y}\right]\right\rangle=\operatorname{deg}(\mathcal{N}),
$$

where "deg" signifies the usual degree of a line bundle on a commutative curve. 
Proof. We can compute as follows,

$$
\begin{aligned}
\operatorname{deg}(\mathcal{N}) & =-\operatorname{deg}\left(\mathcal{N}^{-1}\right) \\
& =-\operatorname{deg}\left(\tau_{*}^{-1}\left(\mathcal{O}_{Y}\right) \otimes_{\mathcal{O}_{Y}} \mathcal{N}^{-1}\right) \\
& \stackrel{(a)}{=}-\operatorname{deg}\left(\mathcal{O}_{Y}(-Y)\right) \\
& \stackrel{(b)}{=}-\left(\chi\left(\mathcal{O}_{Y}(-Y)\right)-\chi\left(\mathcal{O}_{Y}\right)\right) \\
= & \chi\left(c(Y)\left(\left[\mathcal{O}_{Y}\right]\right)\right) \\
= & \left\langle Y,\left[\mathcal{O}_{Y}\right]\right\rangle,
\end{aligned}
$$

where "(a)" comes from lemma 4.4 s equation (4.4), while "(b)" is valid by the Riemann-Roch theorem for the commutative curve $Y$; see [5, thm. IV.1.3].

In fact, this contains the commutative self intersection theorem for curves on surfaces, [5. ex. V.1.4.1], as a special case; cf. the remarks after theorem 5.2 .

After these facts, we proceed with proving that $\langle-,-\rangle$ has some handy additivity properties.

Lemma 4.6. Let $\left(X, \mathcal{O}_{X}\right)$ be a noetherian quasi-scheme over $k$, and let $Y$ be an effective divisor on $X$. Then for any $M \in \bmod (X)$, we have the equation in $K(X)$,

$$
c\left(o_{X}(-Y)\right)([M])=\left(-c\left(o_{X}(Y)\right)([M])\right)(Y)=(-c(Y)([M]))(Y) .
$$

Proof. For any invertible bi-module $\mathcal{M} \in \operatorname{BIMOD}(X, X)$, we can compute as follows,

$$
\begin{aligned}
c\left(\mathcal{M}^{-1}\right)([M]) & =[M]-[M] \otimes \mathcal{M} \\
& =\left([M] \otimes \mathcal{M}^{-1}-[M]\right) \otimes \mathcal{M} \\
& =-c(\mathcal{M})([M]) \otimes \mathcal{M} .
\end{aligned}
$$

Setting $\mathcal{M}=o_{X}(Y)$ in this formula gives the lemma.

Lemma 4.7. Let $\left(X, \mathcal{O}_{X}\right)$ be a noetherian quasi-scheme over $k$, satisfying condition [Euler], and let $Y$ be an effective divisor on $X$ satisfying condition [Invariant].

$\left.1^{\circ}\right)$ If $M \in \bmod (X)$ is an object of finite length, then

$$
\chi(M(Y))=\chi(M) .
$$

$\left.2^{\circ}\right)$ The homomorphism $\langle Y,-\rangle$ vanishes on $F_{0} K(X)$.

Proof. $1^{\circ}$ : When $M$ has finite length, it has a composition series with simple quotients $q_{1}, \ldots, q_{s}$. Since $-\otimes o_{X}(Y)$ is an equivalence of categories, $M(Y)$ has a composition series with simple quotients $q_{1}(Y), \ldots, q_{s}(Y)$. And by condition [Invariant], we get the second "=" in

$$
\chi(M)=\sum_{i=1}^{s} \chi\left(q_{i}\right)=\sum_{i=1}^{s} \chi\left(q_{i}(Y)\right)=\chi(M(Y)) .
$$

$2^{\circ}$ : Let $[M]$ be a generator of $F_{0} K(X)$. So $M$ is noetherian of Krull dimension 0 , hence of finite length. Using $1^{\circ}$, we get

$$
\begin{aligned}
\langle Y,[M]\rangle & =\chi(c(Y)([M])) \\
& =\chi(M)-\chi(M(-Y)) \\
& =\chi(M(-Y)(Y))-\chi(M(-Y)) \\
& =0 .
\end{aligned}
$$


Theorem 4.8. Let $\left(X, \mathcal{O}_{X}\right)$ be a noetherian quasi-scheme over $k$, satisfying condition [Euler], and let $Y, Z, W$ be effective divisors on $X$ such that

- $Y$ satisfies condition [Invariant].

- $Z$ satisfies condition [Fixd comp].

- $W$ satisfies condition [1-dim].

Then for any $z \in K(X)$, and any integers $m, n$ (positive or negative), we have $\left\langle Y, c\left(o_{X}(m Z) \otimes o_{X}(n W)\right)(z)\right\rangle=\left\langle Y, c\left(o_{X}(m Z)\right)(z)\right\rangle+\left\langle Y, c\left(o_{X}(n W)\right)(z)\right\rangle$.

Proof. We have

$$
\begin{aligned}
& \left\langle Y, c\left(o_{X}(m Z)\right)(z)\right\rangle+\left\langle Y, c\left(o_{X}(n W)\right)(z)\right\rangle \\
& -\left\langle Y, c\left(o_{X}(m Z) \otimes o_{X}(n W)\right)(z)\right\rangle \\
& =\left\langle Y,\left(c\left(o_{X}(m Z)\right)+c\left(o_{X}(n W)\right)-c\left(o_{X}(m Z) \otimes o_{X}(n W)\right)\right)(z)\right\rangle \\
& \stackrel{(a)}{=}\left\langle Y, c\left(o_{X}(m Z)\right) \circ c\left(o_{X}(n W)\right)(z)\right\rangle \\
& =(*) \text {, }
\end{aligned}
$$

where " $(a)$ " follows from lemma 3.5 We want to see that $(*)=0$. The idea of the proof is to see that $c\left(o_{X}(n W)\right)$ maps $K(X)$ into $F_{1} K(X)$, and that $c\left(o_{X}(m Z)\right)$ maps $F_{1} K(X)$ into $F_{0} K(X)$; it will then follow from lemma 4.7. part $2^{\circ}$, that $(*)=0$, since $Y$ satisfies condition [Invariant].

We start by proving that

$$
c\left(o_{X}(n W)\right)(K(X)) \subseteq F_{1} K(X) .
$$

There are three cases:

Case 1: $n=0$. Since $o_{X}(0 W)=o_{X}$, and $c\left(o_{X}\right)=0$, equation (4.7) is trivial in this case.

Case 2: $n \geq 1$. Since $W$ satisfies condition [1-dim], so does $n W$ by proposition 2.4. So $n W$ is 1-dimensional, and by lemma 3.4, part $1^{\circ}$, the endomorphism $c\left(o_{X}(n W)\right)$ maps into the image of the canonical map $K(n W) \longrightarrow K(X)$. But then clearly, $c\left(o_{X}(n W)\right)$ maps into $F_{1} K(X)$, and we have proved equation (4.7).

Case 3: $n \leq-1$. By lemma 4.6, we have for any $z \in K(X)$ that

$$
c\left(o_{X}(n W)\right)(z)=-\left(c\left(o_{X}(-n W)\right)(z)\right) \otimes o_{X}(-n W)=(* *) .
$$

The integer $-n$ is positive, so by the argument given for case 2 , we have

$$
c\left(o_{X}(-n W)\right)(z) \in F_{1} K(X) .
$$

So clearly also $(* *) \in F_{1} K(X)$, and we have proved equation (4.7).

We go on to prove that

$$
c\left(o_{X}(m Z)\right)\left(F_{1} K(X)\right) \subseteq F_{0} K(X) .
$$

We again split into three cases:

Case 1: $m=0$. Since $o_{X}(0 Z)=o_{X}$, and $c\left(o_{X}\right)=0$, equation (4.8) is trivial in this case.

Case 2: $m \geq 1$. Since $Z$ satisfies condition [Fixd comp], so does $m Z$ by proposition 2.6 But then lemma 3.4, part $2^{\circ}$, states directly that equation (4.8) is valid.

Case 3: $m \leq-1$. By lemma 4.6, we have for any $z \in F_{1} K(X)$ that

$$
c\left(o_{X}(m Z)\right)(z)=-\left(c\left(o_{X}(-m Z)\right)(z)\right) \otimes o_{X}(-m Z)=(* * *) .
$$

The integer $-m$ is positive, so by the argument given for case 2 , we have

$$
c\left(o_{X}(-m Z)\right)(z) \in F_{0} K(X) .
$$


So clearly also $(* * *) \in F_{0} K(X)$, and we have proved equation (4.8).

Combining equations (4.7) and (4.8), we have that

$$
c\left(o_{X}(m Z)\right) \circ c\left(o_{X}(n W)\right)(K(X)) \subseteq F_{0} K(X) .
$$

In conjunction with lemma 4.7 part $2^{\circ}$, this implies

$$
(*)=0 \text {, }
$$

as desired.

Corollary 4.9. Let $\left(X, \mathcal{O}_{X}\right)$ be a noetherian quasi-scheme over $k$, satisfying condition [Euler], and let $Y, Z$ be effective divisors on $X$ such that

- $Y$ satisfies condition [Invariant].

- $Z$ satisfies conditions [1-dim] and [Fixd comp].

For each integer $m$ (positive or negative) and each $z \in K(X)$, we have

$$
\left\langle Y, c\left(o_{X}(m Z)\right)(z)\right\rangle=m\langle Y, c(Z)(z)\rangle .
$$

Proof. The case $m \geq 1$ is immediate from theorem 4.8 by induction. The case $m=0$ is trivial. And if $m \leq-1$, we note that

$$
o_{X}(m Z) \otimes o_{X}(-m Z)=o_{X}
$$

whence

$$
\begin{aligned}
0 & =\langle Y, 0\rangle \\
& =\left\langle Y, c\left(o_{X}\right)(z)\right\rangle \\
& =\left\langle Y, c\left(o_{X}(m Z) \otimes o_{X}(-m Z)\right)(z)\right\rangle \\
& =\left\langle Y, c\left(o_{X}(m Z)\right)(z)\right\rangle+\left\langle Y, c\left(o_{X}(-m Z)\right)(z)\right\rangle,
\end{aligned}
$$

where the last "=" uses theorem 4.8, Combining this with the result for $m \geq 1$, we get the corollary's statement.

Theorem 4.10. Let $\left(X, \mathcal{O}_{X}\right)$ be a noetherian quasi-scheme over $k$, satisfying condition [Euler], let $z \in F_{1} K(X)$ (note that $z$ has to be in $F_{1} K(X)$ !), and let $Y, Z$ be effective divisors on $X$ such that

- $Y$ satisfies condition [Invariant].

- $Z$ satisfies condition [Fixd comp].

Let $W$ be the effective divisor on $X$ determined by the invertible subobject $o_{X}(-Z) \otimes o_{X}(-Y)$ in $o_{X}$. Then

$$
\langle W, z\rangle=\langle Y, z\rangle+\langle Z, z\rangle .
$$

Proof. We may compute as follows, using $o_{X}(-W)=o_{X}(-Z) \otimes o_{X}(-Y)$ :

$$
\begin{aligned}
(\langle Y, z\rangle+\langle Z, z\rangle)-\langle W, z\rangle & \\
= & \chi\left(\left(c\left(o_{X}(Y)\right)+c\left(o_{X}(Z)\right)-c\left(o_{X}(W)\right)\right)(z)\right) \\
= & \chi\left(\left(c\left(o_{X}(-Y)^{-1}\right)+c\left(o_{X}(-Z)^{-1}\right)\right.\right. \\
& \left.\left.-c\left(o_{X}(-Y)^{-1} \otimes o_{X}(-Z)^{-1}\right)\right)(z)\right) \\
\stackrel{(a)}{=} & \chi\left(c\left(o_{X}(-Y)^{-1}\right) \circ c\left(o_{X}(-Z)^{-1}\right)(z)\right) \\
= & \chi\left(c\left(o_{X}(Y)\right) \circ c\left(o_{X}(Z)\right)(z)\right) \\
= & \langle Y, c(Z)(z)\rangle \\
= & (*)
\end{aligned}
$$


where " $(a)$ " comes from lemma 3.5. To see that $(*)=0$, we observe that since $z \in F_{1} K(X)$, we have $c(Z)(z) \in F_{0} K(X)$ by lemma 3.4, part $2^{\circ}$, and condition [Fixd comp] for $Z$. And hence $(*)=0$ by lemma 4.7 part $2^{\circ}$, and condition [Invariant] for $Y$.

Corollary 4.11. Let $\left(X, \mathcal{O}_{X}\right)$ be a noetherian quasi-scheme over $k$, satisfying condition [Euler], let $z \in F_{1} K(X)$, and let $Y$ be an effective divisor on $X$ which satisfies conditions [Invariant] and [Fixd comp].

Then for each $n \geq 0$, we have

$$
\langle n Y, z\rangle=n\langle Y, z\rangle .
$$

Proof. The case $n=0$ is obvious. And the case $n \geq 1$ follows immediately from theorem 4.10 by induction.

\section{The Riemann-Roch theOREM}

We remind the reader that the integer $d$ from definition 1.14 is assumed to be 2 .

This section gives our main results, theorems 5.1 and 5.2 These are the noncommutative generalizations of the Riemann-Roch theorem and the genus formula for curves on surfaces. The results contain their commutative counterparts for projective surfaces as special cases.

Having obtained these results, we apply them to investigate the behaviour of the quantity $\chi\left(\mathcal{O}_{X}(n Y)\right)$. It turns out to be a second degree polynomial in $n$ which we call $\mathcal{O}_{X}$ 's Hilbert polynomial. We give explicit expressions for the coefficients of the Hilbert polynomial in corollaries 5.3 and 5.4. These expressions are just like the ones encountered in commutative geometry. The use of the first of these expressions is illustrated in example $\mathbf{5 . 5}$, which computes the degree of a certain normal bundle.

The remaining part of the section considers the quantity $\chi(M(n Y))$ for an arbitrary $M \in \bmod (X)$. The main results are theorem 5.6 and corollary 5.9, which tell us that $\chi(M(n Y))$ is a polynomial in $n$ with various properties.

Theorem 5.1 (Riemann-Roch). Let $\left(X, \mathcal{O}_{X}\right)$ be a noetherian quasi-scheme over $k$, satisfying conditions [Euler] and [Dual], and let $Y$ be an effective divisor on $X$ satisfying conditions [1-dim], [Admissible], [Fixd comp], and [Invariant].

Then there is the formula

$$
\chi\left(\mathcal{O}_{X}(Y)\right)=\chi\left(\mathcal{O}_{X}\right)+\frac{1}{2}\left(\left\langle Y,\left[\mathcal{O}_{Y}\right]\right\rangle-\left\langle Y,[\omega]-\left[\mathcal{O}_{X}\right]\right\rangle\right)
$$

(recall that $\omega \in \bmod (X)$ is the dualizing object which exists by condition [Dual]).

Proof. Note first that by duality,

$$
\chi(\omega)=\chi\left(\mathcal{O}_{X}\right),
$$


and

$$
\begin{aligned}
\chi(\omega(-Y)) & =\sum_{j}(-1)^{j} \operatorname{dim}_{k} \operatorname{Ext}_{X}^{j}\left(\mathcal{O}_{X}, \omega(-Y)\right) \\
& =\sum_{j}(-1)^{j} \operatorname{dim}_{k} \operatorname{Ext}_{X}^{j}\left(\mathcal{O}_{X}(Y), \omega\right) \\
& =\sum_{j}(-1)^{j} \operatorname{dim}_{k} \operatorname{Ext}_{X}^{d-j}\left(\mathcal{O}_{X}, \mathcal{O}_{X}(Y)\right)^{\prime} \\
& =\chi\left(\mathcal{O}_{X}(Y)\right) .
\end{aligned}
$$

We can now compute as follows:

$$
\begin{aligned}
& \left\langle Y,\left[\mathcal{O}_{Y}\right]\right\rangle-\left\langle Y,[\omega]-\left[\mathcal{O}_{X}\right]\right\rangle \\
& \stackrel{(a)}{=}\left\langle Y, c\left(o_{X}(Y)\right)\left(\left[\mathcal{O}_{X}\right]\right)\right\rangle-\left\langle Y,[\omega]-\left[\mathcal{O}_{X}\right]\right\rangle \\
& \stackrel{(b)}{=}-\left\langle Y, c\left(o_{X}(-Y)\right)\left(\left[\mathcal{O}_{X}\right]\right)\right\rangle-\left\langle Y,[\omega]-\left[\mathcal{O}_{X}\right]\right\rangle \\
& =-\left\langle Y,\left[\mathcal{O}_{X}\right]-\left[\mathcal{O}_{X}(Y)\right]\right\rangle-\left\langle Y,[\omega]-\left[\mathcal{O}_{X}\right]\right\rangle \\
& =\left\langle Y,\left[\mathcal{O}_{X}(Y)\right]-[\omega]\right\rangle \\
& =\chi\left(c\left(o_{X}(Y)\right)\left(\left[\mathcal{O}_{X}(Y)\right]-[\omega]\right)\right) \\
& =\chi\left(\left[\mathcal{O}_{X}(Y)\right]-[\omega]-\left[\mathcal{O}_{X}\right]+[\omega(-Y)]\right) \\
& \stackrel{(c)}{=} 2\left(\chi\left(\mathcal{O}_{X}(Y)\right)-\chi\left(\mathcal{O}_{X}\right)\right),
\end{aligned}
$$

where " $(a)$ " is by example 3.3, "(b)" is by corollary 4.9, and " $(c)$ " is by equations (5.1) and (5.2). Rearranging terms produces the equation displayed in the theorem.

Theorem 5.2 (The genus formula). Let $\left(X, \mathcal{O}_{X}\right)$ be a noetherian quasi-scheme over $k$, satisfying conditions [Euler] and [Dual], and let $Y$ be an effective divisor on $X$ satisfying conditions [1-dim], [Admissible], [Fixd comp], and [Invariant].

Set $g(Y)=1-\chi\left(\mathcal{O}_{Y}\right)$ (note that if $k$ is algebraically closed, and $Y$ is a commutative smooth projective curve, then $g(Y)$ is the usual genus of $Y$ ).

Then there is the formula

$$
2 g(Y)-2=\left\langle Y,\left[\mathcal{O}_{Y}\right]\right\rangle+\left\langle Y,[\omega]-\left[\mathcal{O}_{X}\right]\right\rangle .
$$

Proof. First we compute

$$
\begin{aligned}
& \left\langle Y,[\omega]-\left[\mathcal{O}_{X}\right]\right\rangle \\
& =\chi\left(c\left(o_{X}(Y)\right)\left([\omega]-\left[\mathcal{O}_{X}\right]\right)\right) \\
& =\chi\left([\omega]-\left[\mathcal{O}_{X}\right]-[\omega(-Y)]+\left[\mathcal{O}_{X}(-Y)\right]\right) \\
& =\chi\left(\mathcal{O}_{X}(-Y)\right)-\chi(\omega(-Y)) \\
& =\chi\left(\mathcal{O}_{X}(-Y)\right)-\chi\left(\mathcal{O}_{X}(Y)\right),
\end{aligned}
$$

where the latter two equalities follow from equations (5.1) and (5.2). 
Now,

$$
\begin{aligned}
& \left\langle Y,\left[\mathcal{O}_{Y}\right]\right\rangle+\left\langle Y,[\omega]-\left[\mathcal{O}_{X}\right]\right\rangle \\
& \quad=\left(\left\langle Y,\left[\mathcal{O}_{Y}\right]\right\rangle-\left\langle Y,[\omega]-\left[\mathcal{O}_{X}\right]\right\rangle\right)+2\left\langle Y,[\omega]-\left[\mathcal{O}_{X}\right]\right\rangle \\
& \stackrel{(a)}{=} 2\left(\chi\left(\mathcal{O}_{X}(Y)\right)-\chi\left(\mathcal{O}_{X}\right)\right)+2\left\langle Y,[\omega]-\left[\mathcal{O}_{X}\right]\right\rangle \\
& \stackrel{(b)}{=} 2\left(\chi\left(\mathcal{O}_{X}(Y)\right)-\chi\left(\mathcal{O}_{X}\right)\right)+2\left(\chi\left(\mathcal{O}_{X}(-Y)\right)-\chi\left(\mathcal{O}_{X}(Y)\right)\right) \\
& =2\left(\chi\left(\mathcal{O}_{X}(-Y)\right)-\chi\left(\mathcal{O}_{X}\right)\right) \\
& =-2 \chi\left(c(Y)\left(\left[\mathcal{O}_{X}\right]\right)\right) \\
& \stackrel{(c)}{=}-2 \chi\left(\left[\mathcal{O}_{Y}\right]\right) \\
& =2 g(Y)-2 .
\end{aligned}
$$

Here " $(a)$ " follows from the Riemann-Roch theorem, "(b)" follows from equation (5.3), and " $(c)$ " follows from example 3.3

Theorems 5.1 and 5.2 contain the commutative Riemann-Roch theorem and genus formula as special cases (although one has to restrict Riemann-Roch to deal with effective divisors): Suppose that $k$ is algebraically closed, and that one has a commutative smooth projective surface $X$ over $k$, and an effective divisor $Y$ on $X$. As remarked in section $1\left(\operatorname{Mod}(X), \mathcal{O}_{X}\right)$ is then a noetherian quasi-scheme over $k$, and $Y$ determines an effective divisor on the quasi-scheme $X$ in the sense of definition 1.3 .

It is classical that $\left(X, \mathcal{O}_{X}\right)$ satisfies conditions [Euler] (see [5, thms. III.2.7 and III.5.2]) and [Dual] (see [5, thm. III.7.6]).

It is easy to verify that $Y$ satisfies conditions [1-dim] (clear, since $Y$ is a curve), [Admissible] (clear, from the commutative definition of effective divisors as invertible ideal sheaves), [Fixd comp] (follows since $-\otimes o_{X}(Y)$ is given by tensoring with a locally free sheaf of rank one, which does not move generic points), and [Invariant] (also follows since $-\otimes o_{X}(Y)$ is given by tensoring with a locally free sheaf of rank one).

So all conditions in theorems 5.1 and 5.2 hold, and we now claim that the statements in the theorems really are the usual Riemann-Roch theorem and genus formula for $Y$. For this, one has to note two things:

$1^{\circ}$ ) If $C$ is a 1 -dimensional module on $X$, then $\langle Y,[C]\rangle=Y . C$, where $Y . C$ is the classical intersection multiplicity between $Y$ and $C$, as defined in [5, sec. V.1]. This is easy to check, using the definition of $\langle Y,-\rangle$ (definition 4.1), and [5, exer. V.1.1].

$\left.2^{\circ}\right)$ We have $\left\langle Y,[\omega]-\left[\mathcal{O}_{X}\right]\right\rangle=Y . K$, where $K$ is the canonical divisor on $X$. This is also easy to check, since we have in $K$-theory that $[K]=[\omega]-\left[\mathcal{O}_{X}\right]$, whence

$$
\left\langle Y,[\omega]-\left[\mathcal{O}_{X}\right]\right\rangle=\langle Y,[K]\rangle=Y . K,
$$

where the second "=" comes from $1^{\circ}$. (Note that $[K]$ is not necessarily equal to $[C]$ for a single 1-dimensional module $C$, but $[K]$ is a linear combination of classes of the form $[C]$, so $1^{\circ}$ applies to $K$.)

Using $1^{\circ}$ and $2^{\circ}$, we can rewrite theorem 5.1 as

$$
\chi\left(\mathcal{O}_{X}(Y)\right)=\chi\left(\mathcal{O}_{X}\right)+\frac{1}{2} Y .(Y-K),
$$


reproducing classical Riemann-Roch for the effective divisor $Y$ (see [5, thm. V.1.6]), and we can rewrite theorem 5.2 as

$$
2 g(Y)-2=Y .(Y+K)
$$

reproducing the classical genus formula (see [5] prop. V.1.5]).

We can embellish the non-commutative Riemann-Roch theorem in the following obvious way:

Corollary 5.3 (Hilbert polynomial I: A formula for $\left.\chi\left(\mathcal{O}_{X}(n Y)\right)\right)$. Let $\left(X, \mathcal{O}_{X}\right)$ be a noetherian quasi-scheme over $k$, satisfying conditions [Euler] and [Dual $+\mathrm{K}]$, and let $Y$ be an effective divisor on $X$ satisfying conditions [1-dim], [Admissible], [Fixd comp], and [Invariant].

Then for each $n \geq 0$, we have

$$
\chi\left(\mathcal{O}_{X}(n Y)\right)=\frac{1}{2}\left\langle Y,\left[\mathcal{O}_{Y}\right]\right\rangle n^{2}-\frac{1}{2}\left\langle Y,[\omega]-\left[\mathcal{O}_{X}\right]\right\rangle n+\chi\left(\mathcal{O}_{X}\right) .
$$

Remark. As we shall see in corollary [5.7, the formula from corollary 5.3 is in fact valid for any integer $n$.

Proof. The corollary's formula is obvious for $n=0$, so we assume that $n \geq 1$. By remark 2.7, the effective divisor $n Y$ satisfies conditions [1-dim], [Admissible], and [Fixd comp], and it is obvious that it satisfies [Invariant]. So we may apply the Riemann-Roch theorem to it, getting

$$
\begin{aligned}
& \chi\left(\mathcal{O}_{X}(n Y)\right) \\
& =\chi\left(\mathcal{O}_{X}\right)+\frac{1}{2}\left(\left\langle n Y,\left[\mathcal{O}_{n Y}\right]\right\rangle-\left\langle n Y,[\omega]-\left[\mathcal{O}_{X}\right]\right\rangle\right) \\
& \stackrel{(a)}{=} \chi\left(\mathcal{O}_{X}\right) \\
& \quad+\frac{1}{2}\left(\left\langle n Y, n\left[\mathcal{O}_{Y}\right]+\text { term from } F_{0} K(X)\right\rangle-\left\langle n Y,[\omega]-\left[\mathcal{O}_{X}\right]\right\rangle\right) \\
& \stackrel{(b)}{=} \chi\left(\mathcal{O}_{X}\right)+\frac{1}{2}\left(\left\langle n Y,\left[\mathcal{O}_{Y}\right]\right\rangle n-\left\langle n Y,[\omega]-\left[\mathcal{O}_{X}\right]\right\rangle\right) \\
& \stackrel{(c)}{=} \chi\left(\mathcal{O}_{X}\right)+\frac{1}{2}\left(\left\langle Y,\left[\mathcal{O}_{Y}\right]\right\rangle n^{2}-\left\langle Y,[\omega]-\left[\mathcal{O}_{X}\right]\right\rangle n\right)
\end{aligned}
$$

and this is the desired formula. Here " $(a)$ " is by lemma 2.8, "(b)" is by lemma 4.7 part $2^{\circ}$, and "(c)" is by corollary 4.11 .

Note that it is the application of corollary 4.11 which makes it necessary to assume condition $[$ Dual $+\mathrm{K}]$ rather than just [Dual]: Condition [Dual $+\mathrm{K}]$ requires that $[\omega]-\left[\mathcal{O}_{X}\right] \in F_{1} K(X)$, and this is needed for the application of corollary 4.11

As a variation of this corollary, we have

Corollary 5.4 (Hilbert polynomial II: Another formula for $\left.\chi\left(\mathcal{O}_{X}(n Y)\right)\right)$. Let $(X$, $\left.\mathcal{O}_{X}\right)$ be a quasi-scheme over $k$, satisfying conditions [Euler] and [Dual $\left.+\mathrm{K}\right]$, and let $Y$ be an effective divisor on $X$ satisfying conditions [1-dim], [Admissible], [Fixd comp], and [Invariant].

Then for each $n \geq 0$, we have

$$
\chi\left(\mathcal{O}_{X}(n Y)\right)=\frac{1}{2}\left\langle Y,\left[\mathcal{O}_{Y}\right]\right\rangle n^{2}+\left(\frac{1}{2}\left\langle Y,\left[\mathcal{O}_{Y}\right]\right\rangle+1-g(Y)\right) n+\chi\left(\mathcal{O}_{X}\right)
$$

(this is the same formula as in the commutative case; see [5, exer. V.1.2]). 
Remark. As we shall see in corollary [5.7, the formula from corollary [5.4 is in fact valid for any integer $n$.

Proof. This is obtained by combining corollary 5.3 with theorem 5.2

$$
\begin{aligned}
\chi\left(\mathcal{O}_{X}(n Y)\right) & =\frac{1}{2}\left\langle Y,\left[\mathcal{O}_{Y}\right]\right\rangle n^{2}-\frac{1}{2}\left\langle Y,[\omega]-\left[\mathcal{O}_{X}\right]\right\rangle n+\chi\left(\mathcal{O}_{X}\right) \\
& =\frac{1}{2}\left\langle Y,\left[\mathcal{O}_{Y}\right]\right\rangle n^{2}+\left(\frac{1}{2}\left\langle Y,\left[\mathcal{O}_{Y}\right]\right\rangle+1-g(Y)\right) n+\chi\left(\mathcal{O}_{X}\right) .
\end{aligned}
$$

As one can see from theorem 5.2 and corollary $\left[5.4\right.$, the two numbers $\left\langle Y,\left[\mathcal{O}_{Y}\right]\right\rangle$ ("the self intersection") and $g(Y)$ ("the genus") for an effective divisor $Y$ on $X$ are important numerical invariants of $Y$ and its embedding in $X$, just as in commutative geometry.

The next example shows an application of our machinery, in particular, corollary 5.3 to a practical computation.

Example 5.5. (The degree of the normal bundle of the cubic divisor in the elliptic quantum plane.) This example is a continuation of example 1.10, and uses its setup and notation. In particular, $A$ is a graded Artin-Schelter regular algebra of dimension three over the algebraically closed field $k$, and $g \in A_{3}$ is a regular central element. $S=\operatorname{Proj}(A)$ is an elliptic quantum plane, and $T$ is the elliptic curve which is an effective divisor on $S$.

Applying the theory developed above, in particular, theorem 4.5 and corollary 5.3 we will see that it becomes an easy computation to show that the degree of the normal bundle of $T$ in $S$ is

$$
\operatorname{deg}(\mathcal{N})=9
$$

(the construction of $\mathcal{N}$ is described prior to lemma 4.4).

We need to do two things:

$1^{\circ}$ : Check that $S$ and $T$ satisfy the conditions posed in theorem 4.5 and corollary 5.3.

$2^{\circ}$ : Perform the mentioned computation based on those results.

$1^{\circ}$ : Condition [Euler] on $S$ : The algebra $A$ is Artin-Schelter regular, in particular, of finite global dimension, so by [2, thm. 7.2(1)] we see that the cohomology functors $H^{j}(S,-)$ vanish from a certain step. And from [2] thm. 8.1(1)] we know that $A$ satisfies [2]'s condition $\chi$, so by [2, thm. 7.4(1)] we see that each $H^{j}(S,-)$ sends noetherian objects to finite dimensional vector spaces. All in all, this shows that $\left(S, \mathcal{O}_{S}\right)$ satisfies condition [Euler].

Condition $[$ Dual $+\mathrm{K}]$ on $S$ : It is an easy computation to see that over $A$ we have

$$
H_{\mathfrak{m}}^{j}(A)^{\prime}=\left\{\begin{array}{ccc}
0 & \text { for } & j \neq 3 \\
A(-3) & \text { for } & j=3
\end{array}\right.
$$

and [12, thm. 4.2] then implies that $\omega=\pi A(-3)$ is a dualizing object satisfying condition [Dual]. To see that the stronger condition $[$ Dual $+\mathrm{K}]$ is also satisfied, we note that the exact sequence

$$
0 \rightarrow \pi A(-3) \stackrel{\pi(g \cdot)}{\longrightarrow} \pi A \longrightarrow(\pi A) /(\pi A(-3)) \rightarrow 0
$$

gives us

$$
[\omega]-\left[\mathcal{O}_{S}\right]=[\pi A(-3)]-[\pi A]=-[(\pi A) /(\pi A(-3))]=(*)
$$


in $K(S)$, and since $\pi A$ is 2-critical, we clearly have $(*) \in F_{1} K(S)$.

Condition [1-dim] on $T$ : This is clear since example 1.10 shows that $\left(T, \mathcal{O}_{T}\right)$ is just a commutative elliptic curve.

Condition [Admissible] on $T$ : For this, we need to see that $\mathcal{O}_{S}=\pi A$ has no subobject from $\bmod (T)$. But this is clear: The objects in $\bmod (T)$ come from modules which are annihilated by $g$, and $g$ is regular on $A$.

Condition [Fixd comp] on $T$ : That $T$ satisfies condition [Fixd comp] can be read in proposition 1.15 .

Condition [Invariant] on $T$ : Let $p \in \bmod (S)$ be a point, i.e. a simple object. We split into two cases:

Case 1: $p \notin \bmod (T)$. In this case, $\lambda_{p} \neq 0$, so since $p$ is simple we have that $\lambda_{p}: p \longrightarrow p(T)$ is an isomorphism. In particular, we must have

$$
\chi(p(T))=\chi(p) .
$$

Case 2: $p \in \bmod (T)$. We have

$$
\chi_{S}(p) \stackrel{(a)}{=} \chi_{T}(p) \stackrel{(b)}{=} 1,
$$

where " $(a)$ " follows from lemma 1.16 and condition [Admissible] on $T$, and where "(b)" is because $T$ is a commutative smooth projective curve over the algebraically closed field $k$.

Now, $\bmod (T)$ is closed under $-\otimes o_{X}(T)$, so $p(T)$ is also a point on $T$. And equation (5.4) is valid for any point on $T$, so

$$
\chi(p(T))=1=\chi(p) .
$$

$2^{\circ}$ : We now have for $n \gg 0$ that

$$
\chi_{S}\left(\mathcal{O}_{S}(n T)\right)=\chi_{\operatorname{tails}(A)}(\pi A(3 n)) \stackrel{(a)}{=} \operatorname{dim}_{k} A_{3 n} \stackrel{(b)}{=} \frac{9}{2} n^{2}+\frac{9}{2} n+1,
$$

where " $(a)$ " holds because $A$ satisfies $\chi$, and where " $(b)$ " is by the formula for $\operatorname{dim} A_{n}$ given in example 1.10 But by the statements proved in $1^{\circ}$, corollary 5.3 applies to the present situation, and tells us that

$$
\chi\left(\mathcal{O}_{S}(n T)\right)=\frac{1}{2}\left\langle T,\left[\mathcal{O}_{T}\right]\right\rangle n^{2}-\frac{1}{2}\left\langle T,[\omega]-\left[\mathcal{O}_{S}\right]\right\rangle n+\chi\left(\mathcal{O}_{S}\right) .
$$

Comparing coefficients in the two expressions for $\chi\left(\mathcal{O}_{S}(n T)\right)$, we get

$$
\begin{aligned}
\left\langle T,\left[\mathcal{O}_{T}\right]\right\rangle & =9, \\
\left\langle T,[\omega]-\left[\mathcal{O}_{S}\right]\right\rangle & =-9, \\
\chi\left(\mathcal{O}_{S}\right) & =1 .
\end{aligned}
$$

Theorem 4.5 also applies to the present situation, and so we get the desired result,

$$
\operatorname{deg}(\mathcal{N})=\left\langle T,\left[\mathcal{O}_{T}\right]\right\rangle=9
$$

Corollaries 5.3 and 5.4 tell us that the quantity $\chi\left(\mathcal{O}_{X}(n Y)\right)$ is a quadratic polynomial in $n$ for $n \geq 0$, and also give us some expressions for the coefficients of this polynomial. More in the abstract, we can consider any $M \in \bmod (X)$, and ask for the behaviour of $\chi(M(n Y))$. 
Theorem 5.6 (Hilbert polynomial III: The general behaviour of $\chi(M(n Y))$ ). Let $\left(X, \mathcal{O}_{X}\right)$ be a noetherian quasi-scheme satisfying condition [Euler], and let $Y$ be an effective divisor on $X$ satisfying conditions [1-dim], [Fixd comp], and [Invariant]. Let $M \in \bmod (X)$.

$\left.0^{\circ}\right)$ If $\operatorname{Kdim}(M)=0$, then for any $n \in \mathbb{Z}$, we have

$$
\chi(M(n Y))=\chi(M) .
$$

$\left.1^{\circ}\right)$ If $\operatorname{Kdim}(M)=1$, then there exists $b \in \mathbb{R}$ such that for any $n \in \mathbb{Z}$, we have

$$
\chi(M(n Y))=b n+\chi(M) .
$$

$\left.2^{\circ}\right)$ If $\operatorname{Kdim}(M) \geq 2$, then there exist $a, b \in \mathbb{R}$ such that for any $n \in \mathbb{Z}$, we have

$$
\chi(M(n Y))=a n^{2}+b n+\chi(M) .
$$

Proof. $0^{\circ}$ : If $M \in \bmod (X)$, and $\operatorname{Kdim}(M)=0$, then $M$ has finite length. Lemma 4.7, part $1^{\circ}$, then implies that for any integer $n$, we have

$$
\chi(M(n Y))=\chi(M) .
$$

$1^{\circ}$ : Euler characteristic is additive, and the category $\bmod (X)$ has critical composition series, so it is clearly sufficient to prove this statement when $M$ is 1-critical. For such an $M$, there are two cases.

Case 1: $M \notin \bmod (Y)$. We have that $\lambda_{M} \neq 0$, hence $\lambda_{M}(-Y) \neq 0$, so by criticality of $M$ and the sequence (1.4) we have $i^{!} M=0$. Twisting the sequence (1.4) by $n Y$, we get

$$
0 \rightarrow M((n-1) Y) \longrightarrow M(n Y) \longrightarrow\left(i^{*} M\right)(n Y) \rightarrow 0,
$$

and telescoping, we get for $n \geq 1$ that

$$
\begin{aligned}
\chi(M(n Y)) & =\chi(M((n-1) Y))+\chi\left(\left(i^{*} M\right)(n Y)\right) \\
& =\cdots \\
& =\chi(M)+\sum_{\ell=1}^{n} \chi\left(\left(i^{*} M\right)(\ell Y)\right) \\
& =\chi(M)+\chi\left(i^{*} M\right) n .
\end{aligned}
$$

For the last "=", we have used that by criticality of $M$ we have

$$
\operatorname{Kdim}\left(i^{*} M\right)=0,
$$

whence by lemma4.7, part $1^{\circ}$,

$$
\chi\left(\left(i^{*} M\right)(\ell Y)\right)=\chi\left(i^{*} M\right)
$$

for any $\ell$.

Telescoping the other way as well, one gets the same formula as above,

$$
\chi(M(n Y))=\chi(M)+\chi\left(i^{*} M\right) n,
$$

so this formula is valid for any $n \in \mathbb{Z}$.

Case 2: $M \in \bmod (Y)$. We use condition [Fixd comp] to write

$$
[M(Y)]=[M]+y
$$


in $K(Y)$, where $y \in F_{0} K(Y)$. We can telescope as follows for $n \geq 1$,

$$
\begin{aligned}
\chi(M(n Y)) & =\chi(M((n-1) Y))+\chi(y((n-1) Y)) \\
& =\cdots \\
& =\chi(M)+\sum_{\ell=0}^{n-1} \chi(y(\ell Y)) \\
& =\chi(M)+\chi(y) n .
\end{aligned}
$$

For the last "=", we have used lemma 4.7 part $1^{\circ}$, by which we have

$$
\chi(y(\ell Y))=\chi(y)
$$

when $y \in F_{0} K(X)$ and $\ell \in \mathbb{Z}$.

Telescoping the other way as well, one gets the same formula as above,

$$
\chi(M(n Y))=\chi(M)+\chi(y) n,
$$

so this formula is valid for any $n \in \mathbb{Z}$.

$2^{\circ}$ : Given $M \in \bmod (X)$ with $\operatorname{Kdim}(M) \geq 2$. By condition [1-dim], we have $\operatorname{Kdim}\left(i^{!} M\right) \leq 1$ and $\operatorname{Kdim}\left(i^{*} M\right) \leq 1$, and parts $0^{\circ}$ and $1^{\circ}$ above can thus be applied to get real numbers $s, t$ such that for any integer $m$, we have

$$
\begin{aligned}
\chi\left(\left(i^{!} M\right)(m Y)\right) & =s m+\chi\left(i^{!} M\right), \\
\chi\left(\left(i^{*} M\right)(m Y)\right) & =t m+\chi\left(i^{*} M\right) .
\end{aligned}
$$

Consider the exact sequence (1.4), and twist it by $n Y$, getting

$$
0 \rightarrow\left(i^{!} M\right)((n-1) Y) \longrightarrow M((n-1) Y) \longrightarrow M(n Y) \longrightarrow\left(i^{*} M\right)(n Y) \rightarrow 0 .
$$

Using this sequence to telescope, we get for $n \geq 1$ that

$$
\begin{aligned}
\chi(M(n Y)) & =\chi(M((n-1) Y))+\chi\left(\left(i^{*} M\right)(n Y)\right)-\chi\left(\left(i^{!} M\right)((n-1) Y)\right) \\
& =\cdots \\
& =\chi(M)+\sum_{\ell=1}^{n} \chi\left(\left(i^{*} M\right)(\ell Y)\right)-\chi\left(\left(i^{!} M\right)((\ell-1) Y)\right) \\
& \stackrel{(a)}{=} \chi(M)+\sum_{\ell=1}^{n} t \ell+\chi\left(i^{*} M\right)-s(\ell-1)-\chi\left(i^{!} M\right) \\
& =\chi(M)+\left(\chi\left(i^{*} M\right)-\chi\left(i^{!} M\right)+\frac{s+t}{2}\right) n+\frac{-s+t}{2} n^{2},
\end{aligned}
$$

where " $(a)$ " uses equations (5.5). Telescoping the other way, one gets the same equation,

$$
\chi(M(n Y))=\chi(M)+\left(\chi\left(i^{*} M\right)-\chi\left(i^{!} M\right)+\frac{s+t}{2}\right) n+\frac{-s+t}{2} n^{2},
$$

which is therefore valid for any $n \in \mathbb{Z}$.

Corollary 5.7. (Hilbert polynomial I, embellished; compare with corollaries 5.3 and 5.4). Let $\left(X, \mathcal{O}_{X}\right)$ be a noetherian quasi-scheme satisfying conditions [Euler] and $[\mathrm{Dual}+\mathrm{K}]$, and let $Y$ be an effective divisor on $X$ satisfying conditions [1-dim], [Admissible], [Fixd comp], and [Invariant].

Then the formulae from corollaries 5.3 and 5.4 are in fact valid for any integer $n$ (and not just for non-negative $n$ 's). 
Proof. Consider the quantity $\chi\left(\mathcal{O}_{X}(n Y)\right)$. On one hand, by theorem [5.6] it is a polynomial in $n$ of degree at most 2. On the other hand, in corollaries 5.3 and 5.4 we have concrete expressions of $\chi\left(\mathcal{O}_{X}(n Y)\right)$ as polynomials of degree at most 2 , for $n \geq 0$. But these statements are certainly only compatible if the concrete expressions from corollaries 5.3 and 5.4 hold for any integer $n$.

We can apply theorem 5.6 in another obvious way, when the divisor $Y$ is ample. Let us recall from [10, def. 3.9.5] the definition of ampleness:

Definition 5.8. Let $\left(X, \mathcal{O}_{X}\right)$ be a noetherian quasi-scheme over $k$, and let $Y$ be an effective divisor on $X$. We say that $Y$ is ample if the following are satisfied for each $M \in \bmod (X)$ and each $n \gg 0$ :

- $M(n Y)$ is generated by global sections, i.e. there is an epimorphism $\mathcal{O}_{X}^{\oplus m} \longrightarrow$ $M(n Y)$.

- When $i \geq 1$ we have $H^{i}(X, M(n Y))=0$.

Let us now look at a noetherian quasi-scheme $\left(X, \mathcal{O}_{X}\right)$ over $k$, and an effective divisor $Y$ on $X$. We get a triple $\left(\bmod (X), \mathcal{O}_{X},-\otimes o_{X}(Y)\right)$ like the ones considered by Artin and Zhang in [2] sec. 4].

Our triple satisfies [2, cond. (H1)], because we have assumed that $\left(X, \mathcal{O}_{X}\right)$ is noetherian (this includes the condition that $\mathcal{O}_{X}$ is a noetherian object). If we assume that $\left(X, \mathcal{O}_{X}\right)$ satisfies condition [Euler], then the triple also satisfies condition 2, cond. (H2)]. Moreover, if we assume that $Y$ is ample, then it is easy to see that the triple satisfies [2, conds. (H3a) and (H3b)], and also satisfies Serre vanishing as defined in [2, conds. (H4) and (H5)]. So assuming that $X$ satisfies condition [Euler] and that $Y$ is ample, we can set $A=H^{0}\left(\mathcal{O}_{X}\right)_{\geq 0}$, and learn:

- $A$ is a graded left-noetherian locally finite $k$-algebra, and satisfies condition $\chi_{1}$, by [2, thm. 4.5(1)].

- There is an equivalence of triples,

$$
\left(\bmod (X), \mathcal{O}_{X},-\otimes o_{X}(Y)\right) \simeq(\operatorname{tails}(A), \pi(A),(-)(1))
$$

by [2, thm. 4.5(1)]. Note that [2]'s functor $\pi: \operatorname{grmod} A \rightarrow \operatorname{tails} A$ can be thought of as a functor $\pi: \operatorname{grmod} A \rightarrow \bmod X$.

- $A$ satisfies condition $\chi$, by [2, cor. 7.6].

Corollary 5.9 (Hilbert polynomial IV: The case of an ample $Y$ ). Let $\left(X, \mathcal{O}_{X}\right)$ be a noetherian quasi-scheme over $k$, satisfying condition [Euler], and let $Y$ be an effective divisor on $X$ satisfying conditions [1-dim], [Fixd comp], and [Invariant]. Suppose also that $Y$ is ample.

We have the corresponding triple $\left(\bmod (X), \mathcal{O}_{X},-\otimes o_{X}(Y)\right)$, and the ensuing algebra $A=H^{0}\left(\mathcal{O}_{X}\right)_{\geq 0}$, and the conclusions drawn after definition 5.8 hold. Moreover:

$\left.1^{\circ}\right)$ The algebra $A$ admits a theory of Hilbert polynomials in the following sense: Let $M \in \operatorname{grmod}(A)$. Then there exists a (necessarily unique) polynomial $p_{M}(t)$, called $M$ 's Hilbert polynomial, satisfying:

- For $n \gg 0$, we have

$$
\operatorname{dim}_{k} M_{n}=p_{M}(n)
$$

where $M_{n}$ is the $n$th graded piece of $M$.

- For any integer $n \in \mathbb{Z}$, we have

$$
\chi((\pi M)(n Y))=p_{M}(n) .
$$


- The degree of $p_{M}(t)$ is less than or equal to $\operatorname{Kdim}(\pi M)$.

$\left.2^{\circ}\right)$ Let $M \in \operatorname{grmod}(A)$ have $\operatorname{Kdim}(\pi(M))=1$; that is, $\pi(M)$ is a curve module. Then the Hilbert polynomial of $M$ is obtained in the following concrete way: For any integer $n \in \mathbb{Z}$, we have

$$
p_{M}(n)=\chi((\pi M)(n Y))=\langle Y,[\pi M]\rangle n+\chi(\pi M) .
$$

Remark. Note in connection with part $2^{\circ}$ that it really says that when we use $Y$ to realize $X$ as projective space by the equivalence

$$
\left(\bmod (X), \mathcal{O}_{X},-\otimes o_{X}(Y)\right) \simeq(\operatorname{tails}(A), \pi(A),(-)(1)),
$$

and take a module $M \in \operatorname{grmod}(A)$ such that $\pi(M)$ is a curve module, then the multiplicity of $M$ is obtained as the intersection multiplicity $\langle Y,[\pi M]\rangle$. This is entirely analogous to the commutative case; see [5, V.1.6.2].

Proof. $1^{\circ}$ : That $\chi((\pi M)(n Y))$ is equal to $p_{M}(n)$ where $p_{M}$ is a polynomial with degree less than or equal to $\operatorname{Kdim}(\pi M)$ can be read in theorem [5.6, because we have identified tails $(A)$ with $\bmod (X)$.

So we just need to see that $\operatorname{dim}_{k} M_{n}=p_{M}(n)$ for $n \gg 0$. However, for $n \gg 0$ we have

$$
\begin{aligned}
p_{M}(n) & =\chi((\pi M)(n Y)) \\
& =\sum_{j}(-1)^{j} \operatorname{dim}_{k} \operatorname{Ext}_{\text {tails }(A)}^{j}(\pi A, \pi M(n)) \\
& =\operatorname{dim}_{k} \operatorname{Hom}_{\text {tails }(A)}(\pi A, \pi M(n)) \\
& =\operatorname{dim}_{k} M_{n},
\end{aligned}
$$

where the last two "="'s hold because $A$ satisfies condition $\chi$.

$2^{\circ}$ : When $\operatorname{Kdim}(\pi M)=1$, we know from part $1^{\circ}$ that there exists an integer $b$ such that

$$
\chi((\pi M)(n Y))=p_{M}(n)=b n+\chi(\pi M)
$$

for any $n \in \mathbb{Z}$. And in fact,

$$
\begin{aligned}
b & =(b \cdot 0+\chi(\pi M))-(b \cdot(-1)+\chi(\pi M)) \\
& =p_{M}(0)-p_{M}(-1) \\
& =\chi(\pi M)-\chi((\pi M)(-Y)) \\
& =\langle Y,[\pi M]\rangle .
\end{aligned}
$$

\section{INTERSECTION MULTIPLICITIES ON A BLOW UP}

Throughout this section, the base field $k$ is algebraically closed.

To exemplify the theory developed above, this section applies it to the quasischeme $\tilde{X}$ obtained by the blow up $\alpha: \widetilde{X} \longrightarrow X$ of the non-commutative surface $X$ in a suitable point $p$. In particular, it investigates the intersection theoretic properties of the exceptional divisor, $E$ (when this divisor exists), and shows the result given in theorem 6.3 below: The self intersection of $E$ is

$$
\left\langle E,\left[\mathcal{O}_{E}\right]\right\rangle=-1 \text {. }
$$

This is a direct generalization of the corresponding property of the exceptional divisor on a commutative blow up; see [5, prop. V.3.2]. We also apply this result 
in example 6.4 to give an example of a surface with an effective divisor which is isomorphic to a commutative $\mathbb{P}^{1}$, but cannot be "blown down": The divisor turns out to have self intersection +1 , and so cannot be obtained as $E$ on any $\widetilde{X}$.

Let us start by setting up some framework. The theory of non-commutative blow up was developed by Van den Bergh in [10], and we will refer back to that paper all the time. In particular, we will take over the notation and assumptions of [10. So we have a quasi-scheme $\left(X, \mathcal{O}_{X}\right)$ over $k$ which contains a commutative projective curve $Y$ as effective divisor. For each point $q \in Y$, we can consider the skyscraper sheaf $\mathcal{O}_{q}$ at $q$; it is an object of $\bmod (Y)$, and hence also of $\bmod (X)$. Since $Y$ is commutative, $q$ also gives a bi-module $o_{q}$ on $Y$, and we have canonical epimorphisms $o_{X} \longrightarrow o_{Y} \longrightarrow o_{q}$, which allow us to also think of $o_{q}$ as a bi-module on $X$.

Like [10], we will make the standing assumption:

Each $\mathcal{O}_{q}$ has finite injective dimension in $\operatorname{Mod}(X)$.

Let $p$ be a fixed point on $Y$. We define bi-modules on $X$,

$$
\begin{array}{rlrl}
m_{p}=\operatorname{Ker}\left(o_{X} \longrightarrow o_{p}\right), & & I_{p}=m_{p} \otimes o_{X}(Y) ; \\
m_{Y, p} & =\operatorname{Ker}\left(o_{Y} \longrightarrow o_{p}\right), & & I_{Y, p}=m_{Y, p} \otimes o_{X}(Y),
\end{array}
$$

and Rees algebras

$$
\mathcal{D}_{p}=o_{X} \oplus I_{p} \oplus I_{p}^{2} \oplus \cdots, \quad \mathcal{D}_{Y, p}=o_{Y} \oplus I_{Y, p} \oplus I_{Y, p}^{2} \oplus \cdots .
$$

These are $\mathbb{N}$-graded algebras over $X$, and there is a canonical epimorphism $\mathcal{D}_{p} \longrightarrow$ $\mathcal{D}_{Y, p}$. The blow up of $X$ in $p$ is defined as

$$
\left(\widetilde{X}, \mathcal{O}_{\widetilde{X}}\right)=\operatorname{Proj}\left(\mathcal{D}_{p}\right)
$$

and the "strict transform" of $Y$ in $\widetilde{X}$ is defined as

$$
\left(\tilde{Y}, \mathcal{O}_{\tilde{Y}}\right)=\operatorname{Proj}\left(\mathcal{D}_{Y, p}\right)
$$

(see [10, sec. 3.8] for a discussion of Proj). The epimorphism $\mathcal{D}_{p} \longrightarrow \mathcal{D}_{Y, p}$ gives a commutative diagram of quasi-schemes over $k$,

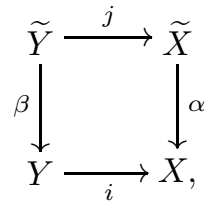

and it turns out by $[10$, thm. $6.3 .1(1)]$ that $\beta: \widetilde{Y} \longrightarrow Y$ is isomorphic to the usual (commutative) blow up of $Y$ in $p$.

Having introduced the blow up, we also want to say something about the exceptional divisor. For this, we define the automorphism $\tau \in \operatorname{Aut}(Y)$ by

$$
\mathcal{O}_{\tau q}=\mathcal{O}_{q}(Y)
$$

for each point $q \in Y$. For the exceptional divisor to exist, it is necessary to suppose that $\tau(p)=p$. In this case, as described in [10, sec. 6.6], $m_{p} \mathcal{D}_{p}$ is an ideal in $\mathcal{D}_{p}$ which is invertible up to right-bounded bi-modules, and so determines an effective 
divisor $E$ on $\widetilde{X}$. Since $o_{p}=o_{X} / m_{p}$ is a quotient of $\mathcal{D}_{p} / m_{p} \mathcal{D}_{p}$, the divisor sits in a commutative diagram of quasi-schemes over $k$,

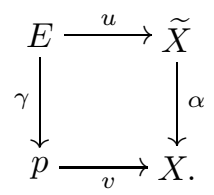

[10, prop. 6.6.2(1)] identifies $E$ with $\mathbb{P}^{1}$, the commutative projective line. Let us describe the identification which we will use below. First note that $\left(E, \mathcal{O}_{E}\right)$ is defined as $\operatorname{Proj}\left(\mathcal{D}_{p} / m_{p} \mathcal{D}_{p}\right)$. Now, [10, proof of 6.6.2(1)] shows that the functor $(-)_{p}^{\wedge}$ introduced in [10, thm. 5.1.4(1)] gives an equivalence

$$
\operatorname{GrMod}\left(\mathcal{D}_{p} / m_{p} \mathcal{D}_{p}\right) \stackrel{\simeq}{\longrightarrow} \operatorname{GrMod}\left(\left(\mathcal{D}_{p} / m_{p} \mathcal{D}_{p}\right)_{p}^{\wedge}\right),
$$

where $A=\left(\mathcal{D}_{p} / m_{p} \mathcal{D}_{p}\right)_{p}^{\wedge}$ is a genuine $\mathbb{N}$-graded $k$-algebra. This gives an equivalence

$$
\left(\bmod (E), \mathcal{O}_{E}\right) \stackrel{\simeq}{\longrightarrow}(\operatorname{tails}(A), \pi(A)) .
$$

And on $\bmod (E)$, the twist $-\otimes o_{\widetilde{X}}(E)$ is given by $((-)(-1)) \otimes o_{X}(Y)$; see [10, proof of 6.6.2(2)]. Translating this to tails $(A)$ by using the compatibilities satisfied by $(-)_{p}^{\wedge}$, we get the autoequivalence

$$
(-)(-1)_{\phi}
$$

where $\phi$ is defined as in [10, thm. 5.1.4(3)].

As indicated in [10 proof of 6.6.2(1)], it turns out that $A$ is either the quantum plane or the Jordan plane, that is, either

$$
A=k\langle x, y\rangle /(y x-q x y) \text { or } A=k\langle x, y\rangle /\left(x y-y x+x^{2}\right),
$$

and so, $A$ is a twist of the commutative polynomial ring $k[x, y]$. This gives an equivalence

$$
(\operatorname{tails}(A), \pi(A)) \stackrel{\simeq}{\longrightarrow}\left(\bmod \left(\mathbb{P}^{1}\right), \mathcal{O}_{\mathbb{P}^{1}}\right) .
$$

And one can easily verify that under this equivalence, the autoequivalence $(-)(-1)_{\phi}$ of tails $(A)$ is sent to an autoequivalence $s$ of $\bmod \left(\mathbb{P}^{1}\right)$ given by $s(-)=((-)(-1)) \circ \sigma_{*}$, where $\sigma$ is an automorphism of $\mathbb{P}^{1}$.

Putting together the two equivalences above produces an equivalence which we denote

$$
\left(\bmod (E), \mathcal{O}_{E}\right) \stackrel{\Psi}{\longrightarrow}\left(\bmod \left(\mathbb{P}^{1}\right), \mathcal{O}_{\mathbb{P}^{1}}\right),
$$

under which the twist $-\otimes o_{\widetilde{X}}(E)$ is sent to the autoequivalence $s(-)=((-)(-1)) \circ$ $\sigma_{*}$. This is the identification of $E$ with $\mathbb{P}^{1}$ that we will work with below.

Finally, in the case $\tau(p)=p$, we also need some information concerning the normal bundle of the exceptional divisor $E$ in $\widetilde{X}$. Recall from the discussion prior to lemma 4.4 that the normal bundle is defined as

$$
\mathcal{N}_{E / \widetilde{X}}=o_{\widetilde{X}}(E) / o_{\widetilde{X}},
$$

and that by lemma 4.4 ,

$$
-\otimes \mathcal{N}_{E / \widetilde{X}}=(-)(E)=-\otimes o_{\widetilde{X}}(E)
$$


as functors on $\operatorname{Mod}(E)$. We can use $\Psi$ to see this as an autoequivalence of $\bmod \left(\mathbb{P}^{1}\right)$. But we already know that $\Psi$ takes $-\otimes o_{\widetilde{X}}(E)$ to the autoequivalence $((-)(-1)) \circ \sigma_{*}$, so we conclude that

$$
\begin{gathered}
\Psi \text { takes }-\otimes \mathcal{N}_{E / \widetilde{X}} \text { to the same autoequivalence, } \\
((-)(-1)) \circ \sigma_{*} .
\end{gathered}
$$

On the other hand, there is a line bundle $\mathcal{N}$ on $\mathbb{P}^{1}$, and an automorphism $\tau$ of $\mathbb{P}^{1}$, such that

$$
\left(-\otimes \mathcal{N}_{E / \widetilde{X}}\right)=\tau_{*}\left(-\otimes \mathcal{O}_{E} \mathcal{N}\right)
$$

as functors on $\bmod \left(\mathbb{P}^{1}\right)$ (see the discussion before lemma 4.4). In this equation, $E$ is identified with $\mathbb{P}^{1}$ via $\Psi$. Combining (6.2) and (6.3), we learn that

$$
((-)(-1)) \circ \sigma_{*}=\tau_{*}\left(-\otimes_{\mathcal{O}_{E}} \mathcal{N}\right) \text {. }
$$

From this, it is easy to see what $\mathcal{N}$ is: The only contribution to $\mathcal{N}$ comes from the shift $(-)(-1)$, whence

$$
\mathcal{N}=\mathcal{O}_{\mathbb{P}^{1}}(-1)
$$

Having performed the above setup, we are ready to do some intersection theory on blow ups. We start by showing that condition [Euler] is inherited from $X$ to $\widetilde{X}$. We reach this goal in corollary 6.2 below.

Proposition 6.1. Consider the above setup (without assuming $\tau(p)=p$ ). For any $M \in \operatorname{Mod}(\tilde{X})$, there is a natural long exact sequence consisting of pieces

$$
\operatorname{Ext}_{X}^{n}\left(\mathcal{O}_{X}, \alpha_{*}(M)\right) \rightarrow \operatorname{Ext}_{\widetilde{X}}^{n}\left(\mathcal{O}_{\widetilde{X}}, M\right) \rightarrow \operatorname{Ext}_{X}^{n-1}\left(\mathcal{O}_{X}, R^{1} \alpha_{*}(M)\right) .
$$

Proof. We first claim that there is a convergent spectral sequence

$$
\operatorname{Ext}_{X}^{p}\left(\mathcal{O}_{X}, R^{q} \alpha_{*}(M)\right) \Longrightarrow \operatorname{Ext}_{\widetilde{X}}^{p+q}\left(\mathcal{O}_{\widetilde{X}}, M\right)
$$

In fact, this is just the Grothendieck spectral sequence for the composition

$$
\operatorname{Hom}_{X}\left(\mathcal{O}_{X},-\right) \circ \alpha_{*}(-)=\operatorname{Hom}_{\widetilde{X}}\left(\mathcal{O}_{\widetilde{X}},-\right) .
$$

So to check the existence of (6.5), we need to see that $\alpha_{*}$ sends injectives to objects which are acyclic for $\operatorname{Hom}_{X}\left(\mathcal{O}_{X},-\right)$, but this follows from [10, lem. 7.2.12].

Now the desired long exact sequence follows immediately from 6.5 , when we note that by [10, thm. $6.3 .1(5)]$, we have $R^{2} \alpha_{*}=R^{3} \alpha_{*}=\cdots=0$.

Corollary 6.2. Consider the above setup (without assuming $\tau(p)=p$ ). If the quasi-scheme $\left(X, \mathcal{O}_{X}\right)$ over $k$ satisfies definition 1.14's condition [Euler], then so does the quasi-scheme $\left(\widetilde{X}, \mathcal{O}_{\widetilde{X}}\right)$ over $k$.

Proof. The functors $R^{i} \alpha_{*}$ send noetherian modules to noetherian modules by 10 . prop. 6.5.2(4)]. So the corollary's statement clearly follows from the long exact sequence given in proposition 6.1

After the above results, which show that the blow up $\widetilde{X}$ possesses certain good properties, we can prove our main result on the exceptional divisor. 
Theorem 6.3. (The self intersection of the exceptional divisor of a blow up) Consider the above setup, and assume that $\tau(p)=p$, so the exceptional divisor $E$ exists. Suppose that the quasi-scheme $\left(X, \mathcal{O}_{X}\right)$ over $k$ satisfies condition [Euler].

Then we have

$$
\left\langle E,\left[\mathcal{O}_{E}\right]\right\rangle=-1
$$

Proof. The noetherian quasi-scheme $\widetilde{X}$ satisfies condition [Euler] by corollary 6.2 And $\left(E, \mathcal{O}_{E}\right)$ can be identified with the commutative smooth projective curve $\mathbb{P}^{1}$. But this situation is treated in theorem 4.5, which states

$$
\left\langle E,\left[\mathcal{O}_{E}\right]\right\rangle=\operatorname{deg}(\mathcal{N}),
$$

where $\mathcal{N}$ is the line-bundle on $\mathbb{P}^{1}$ appearing in equation (6.3). And by equation (6.4) we have $\mathcal{N}=\mathcal{O}_{\mathbb{P}^{1}}(-1)$, whence

$$
\left\langle E,\left[\mathcal{O}_{E}\right]\right\rangle=-1
$$

Example 6.4. (A commutative genus 0 curve which cannot be blown down) Consider the homogenized first Weyl algebra over $k$, defined by

$$
A=\frac{k\langle x, y, z\rangle}{\left(x z-z x, y z-z y, y x-x y-z^{2}\right)} .
$$

It can also be realized as the Ore extension $k[x, z][y ; \delta]$ where $\delta$ is the derivation of $k[x, z]$ determined by $\delta(x)=z^{2}$ and $\delta(z)=0$. This makes it clear that $A$ satisfies the following:

- It is Artin-Schelter regular with $\operatorname{gldim}(A)=3$.

- It has Hilbert series $H_{A}(t)=(1-t)^{-3}$, and consequently, $\operatorname{dim}_{k} A_{n}=\frac{1}{2} n^{2}+$ $\frac{3}{2} n+1$ for any $n \geq 0$.

- It contains the regular central element $z \in A_{1}$, and $A /(z) \cong k[x, y]$. So $\operatorname{Proj}(A /(z))=\left(\mathbb{P}^{1}, \mathcal{O}_{\mathbb{P}^{1}}\right)$.

We know that $\left(S, \mathcal{O}_{S}\right):=\operatorname{Proj}(A)=(\operatorname{Tails}(A), \pi A)$ is a noetherian quasi-scheme over $k$, and as we shall see, $\mathbb{P}^{1}$ is isomorphic to an effective divisor $T$ on $S$. However, using corollary 5.3 we will prove that this divisor has self intersection +1 , and so by theorem 6.3 can not be "blown down".

First we look at the invertible bi-module $o_{S}(-T)$ on $S$ given by the functor $\mathcal{M} \longmapsto \mathcal{M}(1)$. There is a natural transformation

$$
\lambda: \text { id } \longrightarrow \mathcal{H o m}\left(o_{S}(-T),-\right)=(-)(1)
$$

induced by multiplication with the regular central element $z$. This gives a morphism $o_{S}(-T) \longrightarrow o_{S}$ which is in fact monic, as one can see using the same method as in example 1.10, and so determines an effective divisor $T$ on $S$.

By arguments entirely resembling the ones given in examples 1.10 and 5.5 , one can prove the following:

- We have $\left(T, \mathcal{O}_{T}\right)=\operatorname{Proj}(A /(z))=\left(\mathbb{P}^{1}, \mathcal{O}_{\mathbb{P}^{1}}\right)$.

- The quasi-scheme $\left(S, \mathcal{O}_{S}\right)$ over $k$ satisfies conditions [Euler] and [Dual+K].

- The effective divisor $T$ satisfies conditions [1-dim], [Admissible], [Fixd comp], and [Invariant].

The first of these three facts implies that it makes sense to ask: Can $T$ be blown down? That is, does there exist a quasi-scheme $\left(X, \mathcal{O}_{X}\right)$ over $k$ with a suitable point 
$p$, such that when we take the blow up $\widetilde{X} \longrightarrow X$ in $p$, we get $\left(S, \mathcal{O}_{S}\right)=\left(\widetilde{X}, \mathcal{O}_{\widetilde{X}}\right)$, and such that $T$ corresponds to the exceptional divisor $E$ on $\widetilde{X}$ ?

We will use our theory to show that the answer to this question is no. This can be done as follows: We have for $n \gg 0$ that

$$
\chi_{S}\left(\mathcal{O}_{S}(n T)\right)=\chi_{\text {tails }(A)}(\pi A(n)) \stackrel{(a)}{=} \operatorname{dim}_{k} A_{n} \stackrel{(b)}{=} \frac{1}{2} n^{2}+\frac{3}{2} n+1,
$$

where " $(a)$ " is since $A$ satisfies [2]'s condition $\chi$ (see [2, thm. 8.1(1)]), and where "(b)" is by the formula for $\operatorname{dim} A_{n}$ given above. But by the statements on $S$ and $T$ given above, corollary 5.3 applies to the present situation, and tells us that

$$
\chi\left(\mathcal{O}_{S}(n T)\right)=\frac{1}{2}\left\langle T,\left[\mathcal{O}_{T}\right]\right\rangle n^{2}-\frac{1}{2}\left\langle T,[\omega]-\left[\mathcal{O}_{S}\right]\right\rangle n+\chi\left(\mathcal{O}_{S}\right) .
$$

Comparing coefficients in the two expressions for $\chi\left(\mathcal{O}_{S}(n T)\right)$, we get

$$
\left\langle T,\left[\mathcal{O}_{T}\right]\right\rangle=1, \quad\left\langle T,[\omega]-\left[\mathcal{O}_{S}\right]\right\rangle=-3, \quad \chi\left(\mathcal{O}_{S}\right)=1 .
$$

Theorem 6.3 also applies to the present situation, and so we see that $S$ cannot be a blow up, $\widetilde{X}$, in such a way that $T$ is the exceptional divisor: $T$ has self intersection +1 , not -1 .

\section{REFERENCES}

[1] M. Artin, J. Tate, and M. Van den Bergh, Some algebras related to automorphisms of elliptic curves, in "The Grothendieck Festschrift", vol. I, pp. 33-85, Birkhäuser, Boston, 1990. MR 92e: 14002

[2] M. Artin and J. J. Zhang, Noncommutative projective schemes, Adv. Math. 109 (1994), 228-287. MR 96a:14004

[3] W. Fulton, "Intersection Theory", Ergebnisse der Math. und ihrer Grenzgebiete, 3. Folge, Band 2, Springer, Berlin, 1984. MR 85k:14004

[4] P. Gabriel, Des catégories abéliennes, Bull. Math. Soc. France 90 (1962), 323-448. MR 38:1144

[5] R. Hartshorne, "Algebraic Geometry", Grad. Texts in Math. 52, Springer, New York, 1977. MR 57:3116

[6] S. Mac Lane, "Categories for the Working Mathematician", Grad. Texts in Math. 5, Springer, New York, 1971. MR 50:7275

[7] I. Mori and S. P. Smith, Bézout's theorem for quantum $\mathbb{P}^{2}$, preprint, 1997.

[8] I. Mori and S. P. Smith, The Grothendieck group of a quantum projective space bundle, preprint, 1998.

[9] S. P. Smith and J. J. Zhang, Curves on quasi-schemes, Algebras and Represent. Theory 1 (1998), 311-351. CMP 99:11

[10] M. Van den Bergh, Blowing up of non-commutative smooth surfaces, to appear in Mem. Amer. Math. Soc.

[11] M. Van Gastel and M. Van den Bergh, Graded Modules of Gelfand-Kirillov Dimension One over Three-Dimensional Artin-Schelter Regular Algebras, J. Algebra 196 (1997), 251-282. MR 99c: 16020

[12] A. Yekutieli and J. J. Zhang, Serre duality for noncommutative projective schemes, Proc. Amer. Math. Soc. 125 (1997), 697-707. MR 97e:14003

Matematisk Afdeling, Københavns Universitet, Universitetsparken 5, 2100 KøbenHAVN $\varnothing$, DK-DANMARK

E-mail address: popjoerg@math.ku.dk

$U R L$ : www.math.ku.dk/ popjoerg 